\title{
Submilliarcsecond Optical Interferometry of the High-mass X-Ray Binary BP Cru with VLTI/GRAVITY
}

\author{
I. Waisberg ${ }^{1}$, J. Dexter ${ }^{1}$, O. Pfuhl ${ }^{1}$, R. Abuter ${ }^{2}$, A. Amorim ${ }^{3}$, N. Anugu ${ }^{3}$, J. P. Berger ${ }^{2}$, N. Blind ${ }^{4}$, H. Bonnet ${ }^{2}$, W. Brandner ${ }^{5}$, \\ A. Buron ${ }^{1}$, Y. Clénet $^{6}$, W. de Wit ${ }^{7}$, C. Deen ${ }^{1}$, F. Delplancke-Ströbele ${ }^{2}$, R. Dembet ${ }^{6}$, G. Duvert ${ }^{8}$, A. Eckart ${ }^{9,10}$, F. Eisenhauer ${ }^{1}$, \\ P. Fédou ${ }^{6}$, G. Finger ${ }^{2}$, P. Garcia ${ }^{3}$, R. Garcia Lopez ${ }^{5,11}$, E. Gendron ${ }^{6}$, R. Genzel ${ }^{1,12}$, S. Gillessen ${ }^{1}$, X. Haubois ${ }^{7}$, M. Haug ${ }^{1,2}$, \\ F. Haussmann ${ }^{1}$, Th. Henning ${ }^{5}$, S. Hippler ${ }^{5}$, M. Horrobin ${ }^{9}$, Z. Hubert ${ }^{5,6}$, L. Jochum ${ }^{2}$, L. Jocou ${ }^{8}$, P. Kervella ${ }^{6,13}$, Y. Kok ${ }^{1}$, M. Kulas ${ }^{5}$, \\ S. Lacour ${ }^{6}$, V. Lapeyrère ${ }^{6}$, J.-B. Le Bouquin ${ }^{8}$, P. Léna ${ }^{6}$, M. Lippa ${ }^{1}$, A. Mérand ${ }^{2}$, E. Müller ${ }^{2,5}$, T. Ott ${ }^{1}$, L. Pallanca ${ }^{7}$, J. Panduro ${ }^{5}$, \\ T. Paumard ${ }^{6}$, K. Perraut ${ }^{8}$, G. Perrin ${ }^{6}$, S. Rabien ${ }^{1}$, A. Ramírez ${ }^{7}$, J. Ramos ${ }^{5}$, C. Rau ${ }^{1}$, R.-R. Rohloff ${ }^{5}$, G. Rousset ${ }^{6}$, \\ J. Sanchez-Bermudez ${ }^{5}$, S. Scheithauer ${ }^{5}$, M. Schöller ${ }^{2}$, C. Straubmeier ${ }^{9}$, E. Sturm ${ }^{1}$, F. Vincent ${ }^{6}$, I. Wank ${ }^{9}$, E. Wieprecht ${ }^{1}$, M. Wiest $^{9}$, \\ E. Wiezorrek ${ }^{1}$, M. Wittkowski ${ }^{2}$, J. Woillez $^{2}$, and S. Yazici ${ }^{1,9}$ \\ (GRAVITY collaboration) $^{14}$

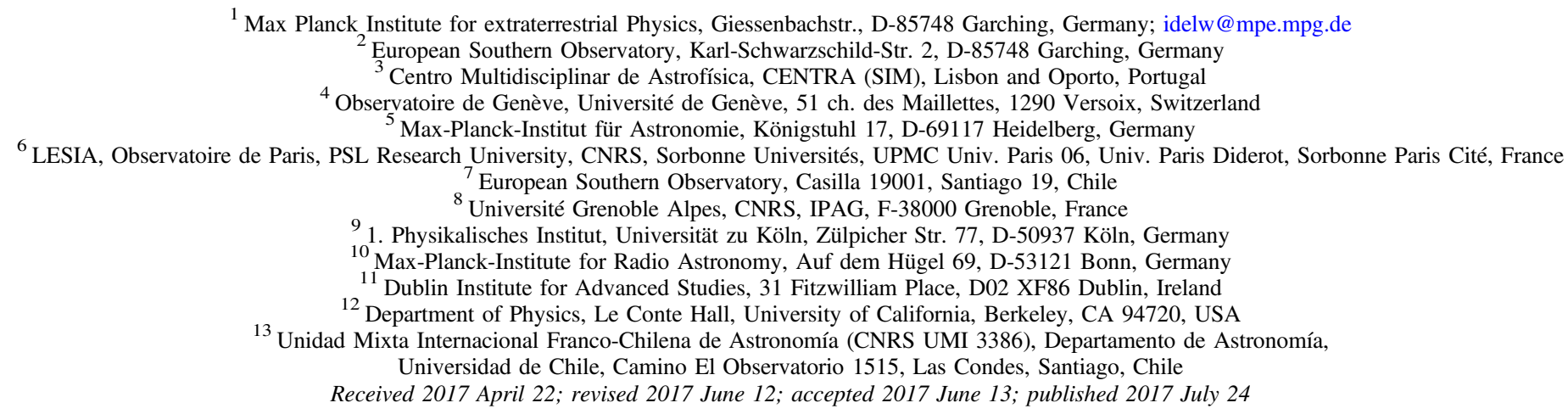

\begin{abstract}
We observe the high-mass X-ray binary (HMXB) BP Cru using interferometry in the near-infrared $K$ band with VLTI/GRAVITY. Continuum visibilities are at most partially resolved, consistent with the predicted size of the hypergiant. Differential visibility amplitude $(\Delta|V| \sim 5 \%)$ and phase $\left(\Delta \phi \sim 2^{\circ}\right)$ signatures are observed across the He I $2.059 \mu \mathrm{m}$ and $\mathrm{Br} \gamma$ lines, the latter seen strongly in emission, unusual for the donor star's spectral type. For a baseline $B \sim 100 \mathrm{~m}$, the differential phase $\mathrm{rms} \sim 0.2$ corresponds to an astrometric precision of $\sim 2 \mu$ as. We generalize expressions for image centroid displacements and variances in the marginally resolved limit of interferometry to spectrally resolved data, and use them to derive model-independent properties of the emission such as its asymmetry, extension, and strong wavelength dependence. We propose geometric models based on an extended and distorted wind and/or a high-density gas stream, which has long been predicted to be present in this system. The observations show that optical interferometry is now able to resolve HMXBs at the spatial scale where accretion takes place, and therefore to probe the effects of the gravitational and radiation fields of the compact object on its environment.
\end{abstract}

Key words: circumstellar matter - techniques: high angular resolution - techniques: interferometric X-rays: binaries - X-rays: individual (GX 301-2)

\section{Introduction}

$\mathrm{X}$-ray binaries are usually divided into two classes: high mass (HMXB), in which the compact object is fed by a strong wind/disk from a massive $\mathrm{OB} / \mathrm{Be}$ companion, and low mass (LMXB), in which accretion happens through Roche lobe overflow from a low-mass star, leading to the formation of an accretion disk around the compact object. In both cases, the compact object can be a white dwarf, neutron star, or a black hole.

\footnotetext{
${ }^{14}$ GRAVITY was developed in a collaboration by the Max Planck Institute for Extraterrestrial Physics, LESIA of Paris Observatory and IPAG of Université Grenoble Alpes/CNRS, the Max Planck Institute for Astronomy, the University of Cologne, the Centro Multidisciplinar de Astrofísica Lisbon and Porto, and the European Southern Observatory.
}

The small scale of such systems, typically with semimajor axis $a<1$ mas, means that they are below the imaging resolution of even the largest optical/near-infrared interferometers. Therefore, information about the accretion process in these systems and the interaction between the compact object's $\mathrm{X}$-ray output and the stellar environment has so far been restricted to X-ray or optical photometry and spectroscopy, from which spatial information are then inferred (for recent reviews on HMXBs in particular, see e.g., Charles \& Coe 2006; Chaty 2011; Walter et al. 2015).

Spectral differential interferometry can provide direct spatial information on scales as small as $\sim 1-10 \mu$ as, depending on the differential visibility precision that can be achieved. However, optical interferometry requires a bright enough object for fringe tracking due to the very short atmospheric coherence time that 
degrades the interferometric signals. For the typical optical/ near-infrared interferometers working in the $V, K$, or $H$ band, this means that nearly all LMXBs and the great majority of HMXBs cannot be observed interferometrically with the current facilities.

GRAVITY (Eisenhauer et al. 2011; Gravity Collaboration et al. 2017), the four-telescope beam combiner working at the Very Large Telescope Interferometer (VLTI) and which operates in the $K$ band, has made it possible to observe fainter objects and to achieve very small differential visibility errors, mainly driven by an improved fringe tracking system that allows for longer coherent integration times, as well as the overall stability of the instrument contributed by its many subsystems. In the case of GRAVITY, fringe tracking limits are $K \lesssim 7$ and $K \lesssim 10$ for the Auxiliary Telescopes (ATs) and Unit Telescopes (UTs), respectively, which means that there are only a handful of Galactic targets that are doable (Liu et al. 2006). We note that dual-field interferometers such as GRAVITY could potentially improve such magnitude limits, provided that a bright enough reference star exists within the field of view (FOV; $2-4$ arcseconds for GRAVITY).

The only published past observations of an HMXB with an optical interferometer were those of Vela X-1 (Choquet et al. 2014) and CI Cam (Thureau et al. 2009 and references therein). The former was observed with VLTI/AMBER in the $K$ band and VLTI/PIONIER in the $H$ band. It contains a supergiant $\mathrm{O}$ star emitting a strong stellar wind and a massive slowly rotating pulsar. Resolved structures of radius $\sim 8 \pm 3 R_{*}$ and $\sim 2 \pm 1 R_{*}$ were inferred from the $K$-and $H$-band continuum visibilities, respectively. Two different interpretations were proposed: the resolved structure could be a stellar wind with a strong temperature gradient that deviates significantly from a blackbody at thermal equilibrium, or the resolved structure in the $K$ band was a diffuse shell not present at the time of the $H$-band observations, which would then correspond to either the stellar wind or the photosphere. Even though spectral lines from $\mathrm{H}$ I and He I were observed in the high-resolution $K$-band spectrum, no differential visibility signatures were detected beyond the noise level, and therefore the application of differential spectral interferometry was not possible. CI Cam was observed with PTI in the $K$ band and with IOTA in the $K$ and $H$ bands. The system is a $\mathrm{B}(\mathrm{e}) \mathrm{X}$-ray binary, and the nature of the compact object is unknown. The interferometric observations were able to resolve extended, hot emission from a ring-shaped circumstellar dust envelope of major axis $\sim 8$ mas. However, no clear evidence for the compact companion was found, and the low resolution did not allow the use of differential spectral interferometry.

$\mathrm{BP} \mathrm{Cru}$ is among the brightest HMXBs in the $K$ band $(K=5.7)$. It is also one of the canonical wind-accreting supergiant HMXBs (Walter et al. 2015); it has, however, several unique properties, some of which are listed in Table 1. It contains a massive and slow-spinning pulsar (GX 301-2) with a typical magnetic field strength of a young neutron star. The donor star, Wray 977, is a rare hypergiant of B1Ia+ classification (Kaper et al. 1995). There are only a handful others in the Galaxy (Clark et al. 2012), and it is the only one known to be in a binary system. Furthermore, it has one of the most eccentric orbits among HMXBs (Liu et al. 2006). It is therefore a promising candidate for studying through optical interferometry the wind and outflow properties of the massive donor star in an HMXB (Martínez-Núñez et al. 2017). With the
Table 1

Properties of BP Cru/Wray 977/GX 301-2

\begin{tabular}{|c|c|c|c|}
\hline Parameter & Symbol/Unit & Value & References \\
\hline & BP Cru & & \\
\hline Distance & $d(\mathrm{kpc})$ & $\approx 3$ & (1) \\
\hline Orbital period & $P_{\text {orb }}$ (days) & $\begin{array}{l}41.498 \\
\quad( \pm 0.002)\end{array}$ & (2) \\
\hline Eccentricity & $e$ & $0.462( \pm 0.014)$ & (2) \\
\hline Binary inclination & $i(\mathrm{deg})$ & $60( \pm 10)$ & (1) \\
\hline $\begin{array}{l}\text { Mean X-ray } \\
\text { luminosity }\end{array}$ & $\left\langle L_{X}\right\rangle\left(\right.$ erg s $\left.^{-1}\right)$ & $7 \times 10^{36}$ & (1) \\
\hline \multirow{2}{*}{$\begin{array}{l}\text { Maximum X-ray } \\
\text { luminosity }\end{array}$} & $L_{X}^{\max }\left(\mathrm{erg} \mathrm{s}^{-1}\right)$ & $4 \times 10^{37}$ & (1) \\
\hline & $\begin{array}{l}\text { Wray } \\
\qquad 977(\mathrm{~B} 1 \mathrm{Ia}+)\end{array}$ & & \\
\hline Mass & $M_{*}\left(M_{\odot}\right)$ & $39-68$ & (1) \\
\hline Radius & $R_{*}\left(R_{\odot}\right)$ & $62^{\mathrm{a}}$ & (1) \\
\hline Photosphere radius & $R_{2 / 3}\left(R_{\odot}\right)$ & $70^{\mathrm{b}}$ & (1) \\
\hline Bolometric luminosity & $L_{*}\left(L_{\odot}\right)$ & $5 \times 10^{5}$ & (1) \\
\hline Effective temperature & $T_{\text {eff }}(K)$ & $18100^{\mathrm{b}}( \pm 500)$ & (1) \\
\hline Mass-loss rate & $\dot{M}\left(M_{\odot} \mathrm{yr}^{-1}\right)$ & $10^{-5}$ & (1) \\
\hline $\begin{array}{l}\text { Wind terminal } \\
\text { velocity }\end{array}$ & $v_{\infty}\left(\mathrm{km} \mathrm{s}^{-1}\right)$ & 305 & (1) \\
\hline $\begin{array}{l}\text { Speed below sonic } \\
\text { point }\end{array}$ & $v_{2 / 3}\left(\mathrm{~km} \mathrm{~s}^{-1}\right)$ & 4.40 & (1) \\
\hline Volume filling factor & $f$ & 1.0 & (1) \\
\hline Rotational velocity & $v \sin i\left(\mathrm{~km} \mathrm{~s}^{-1}\right)$ & $50 \pm 10$ & (1) \\
\hline \multirow{2}{*}{$\begin{array}{l}\text { Radial velocity } \\
\text { amplitude }\end{array}$} & $K_{*}\left(\mathrm{~km} \mathrm{~s}^{-1}\right)$ & $10 \pm 3$ & (1) \\
\hline & GX 301-2 & & \\
\hline $\begin{array}{l}\text { Projected semi- } \\
\text { major axis }\end{array}$ & $a_{X} \sin i(1 \mathrm{t}-\mathrm{s})$ & $368.3 \pm 3.7$ & (2) \\
\hline $\begin{array}{l}\text { Radial velocity } \\
\text { amplitude }\end{array}$ & $K_{X}\left(\mathrm{~km} \mathrm{~s}^{-1}\right)$ & $218.3 \pm 3.3$ & (2) \\
\hline Mass (lower limit) & $M\left(M_{\odot}\right)$ & $1.85 \pm 0.6$ & (1) \\
\hline Spin period & $P_{\text {spin }}(s)$ & 696 & (3) \\
\hline Surface magnetic field & $B(G)$ & $4 \times 10^{12}$ & (3) \\
\hline
\end{tabular}

Notes.

${ }^{\text {a }}$ At Rosseland optical depth $\tau \sim 30$.

${ }^{\mathrm{b}}$ At Rosseland optical depth $\tau=2 / 3$.

References. (1) Kaper et al. (2006); (2) Koh et al. (1997); (3) Kreykenbohm et al. (2004).

goal of studying the inner regions of this system, we have conducted interferometric observations of BP Cru during the commissioning stage of VLTI/GRAVITY in May 2016. This paper reports on these observations.

We summarize the relevant background about this system that will guide us in the interpretation of the interferometric results in Section 2. Section 3 summarizes the observations and the most important aspects of the data reduction. Section 4 presents the analysis of the $K$-band spectrum. Section 5 presents the interferometric results, which are then discussed and fit to physically inspired geometrical models in Section 6. Section 7 presents complementary data that hints at future works for this project. Finally, Section 8 summarizes the main results.

\section{The Effects of the Compact Object on the Surrounding Stellar Environment}

In this section, we summarize relevant information known about BP Cru that will guide the interpretation of the 
Table 2

Summary of Observations

\begin{tabular}{lllc}
\hline \hline Date Time (UTC) & Mode & Integration Time/file & Total Integration Time \\
\hline 2016 May 18 00:56-02:14 & HR COMBINED & DIT $=30$ s NDIT $=10$ & 35 minutes \\
\hline
\end{tabular}

interferometric results. In BP Cru, the pulsar is embedded in the dense stellar wind of Wray 977, and its gravitational and radiation fields are expected to influence the surrounding stellar environment. We note that at the orbital phase of observation ( $\phi \sim 0.21$ using orbital parameters from Koh et al. 1997), the compact object was at a distance $\sim 210 R_{\odot}$ from the donor star's center (the minimum distance at periastron is $\sim 100 R_{\odot}$ ).

\subsection{The Accretion Mechanism and the Gravitational Influence of the Pulsar}

The X-ray emission in wind-accreting HMXBs is explained through the capture of the strong stellar wind of the supergiant companion by the compact object (Bondi \& Hoyle 1944; Davidson \& Ostriker 1973). X-ray light curves and column densities for many of these systems, on the other hand, have found evidence of more complex mechanisms, with a spherically symmetric wind accretion model unable to explain the data successfully.

Stevens (1988) studied the gravitational effects of a compact object on an eccentric orbit in an HMXB system and found that the wind mass-loss rate is substantially enhanced within a small angle around the line of centers, resulting in a higher accretion rate that could explain X-ray outburst intensities better than a spherically symmetric wind accretion model. This inspired accretion models that included, in addition to the spherical wind, a tidal stream of gas of enhanced density that trails the compact object along its orbit and is responsible for most of the accretion rate. In the case of $\mathrm{BP} \mathrm{Cru}$, such models better explain its X-ray emission and column density as a function of orbital phase than purely spherical wind models (Haberl 1991; Leahy 1991, 2002). In particular, the presence of a strong X-ray outburst slightly before periastron, as well as a smaller peak near apastron, could be explained by the pulsar moving through the dense gas stream two times per orbital period. Studies of the $\mathrm{X}$-ray hardness ratio along the orbit are also in rough agreement with such a model (Evangelista et al. 2010). Moreover, an increase in column density during superior conjunction points to a stream of enhanced density trailing the X-ray source. The most recent analysis by Leahy \& Kostka (2008) found a density enhancement in the stream of $\sim 20 \times$ compared to the wind, resulting in a mass-loss rate in the stream $\sim 2.5 \times$ higher than the wind. In this scenario, such a gas stream would then dominate not only the accretion process, but also the mass loss itself. For BP Cru in particular, the high eccentricity, which implies that the pulsar's distance from the massive star varies by a factor of $\frac{1+e}{1-e} \sim 2.7$ (the same holding true for its speed), can lead to complex stream shapes. Kaper et al. (2006) note that tidal interaction is expected during periastron passage, and they also find evidence for variations in the emission and absorption parts of the optical P-Cygni lines $\mathrm{H} \beta$ and $\mathrm{He} \mathrm{I}$ $5876 A$; in particular, a blueshifted absorption component is seen at all orbital phases, which could be evidence for the presence of a large-scale gas stream in the system, both in the orbital plane as well as in the direction perpendicular to it.

Models invoking a circumstellar disk around the supergiant star and inclined with respect to the binary plane have also been proposed as an accretion mechanism (Pravdo et al. 1995). However, they have found less success than the stream models in explaining the X-ray light curve (Leahy 2002). Furthermore, there is no evidence of a circumstellar disk in the optical spectrum (Kaper et al. 2006).

We note that the X-ray light curve of BP Cru is quite stable, with no clear distinction between the low/hard and high/soft states typical of systems containing accretion disks. However, Koh et al. (1997) report on two rapid spin-up episodes of the pulsar lasting for about 30 days, and suggest that this may point to the formation of transient accretion disks following a period of increased accretion rate. Furthermore, the recent, first radio detection from BP Cru suggests a variable component in addition to a baseline component arising from Wray 977's wind, and possibly associated with a weak and transient jet (Pestalozzi et al. 2009).

\subsection{The Radiation Influence of the Pulsar}

The X-ray emission of the pulsar is expected to influence the surrounding stellar environment, mainly through radiation pressure, X-ray heating, and photoionization. In hot stars, the wind is accelerated by scattering from photons absorbed in line transitions (CAK model; Castor et al. 1975). The ionization of the wind results in a cutoff in the wind acceleration, leading to an increase in the wind density that has been evoked to explain the increase in accretion rates in systems that undergo transient behavior. At very high X-ray illumination that suppresses radiative cooling, $\mathrm{X}$-ray heating can lead to thermally driven winds (Blondin 1994).

Haberl (1991) and Islam \& Paul (2014) found evidence for $\mathrm{X}$-ray ionization of the wind when BP Cru was in outburst near periastron from a low energy excess $\lesssim 3 \mathrm{keV}$ in the $\mathrm{X}$-ray spectrum. Variations in the X-ray mean brightness between different orbital periods could also point to X-ray irradiation effects (Leahy \& Kostka 2008). Finally, we note that recently, about two months before the observations reported in this paper, an unusual and extremely bright X-ray outburst was reported with Swift with evidence for strong ionization of the surrounding environment (Fuerst et al. 2016).

In summary, there is ample evidence that the pulsar is closely interacting with the stellar environment in BP Cru. Recent 3D hydrodynamical simulations to study simultaneously the gravitational and radiation effects of the compact object on the stellar wind of HMXBs support that these interactions should play an important role in such systems (Walder et al. 2014; Čechura \& Hadrava 2015).

\section{Observations and Data Reduction}

\subsection{Instrument Setup and Observations}

We have observed BP Cru with VLTI/GRAVITY on the night of 2016 May 18 with the UTs. The observations were carried out in high resolution $(R=4000)$ and in combined (i.e., no split polarization) mode. Table 2 summarizes the observations. Figure 1 shows the corresponding $u v$ coverage. 


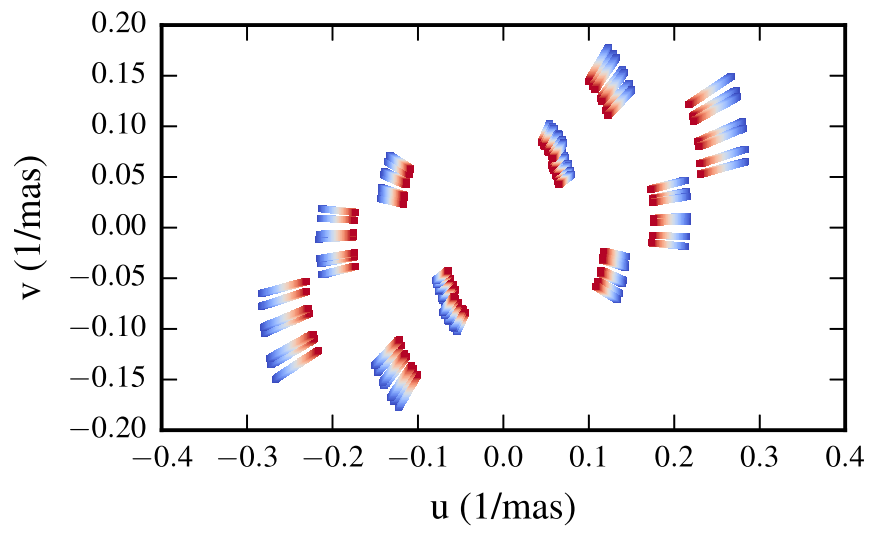

Figure 1. $u v$ coverage of our GRAVITY BP Cru observations. The colors represent the different wavelength channels across the $K$ band, from blue $(1.99 \mu \mathrm{m})$ to red $(2.45 \mu \mathrm{m})$.

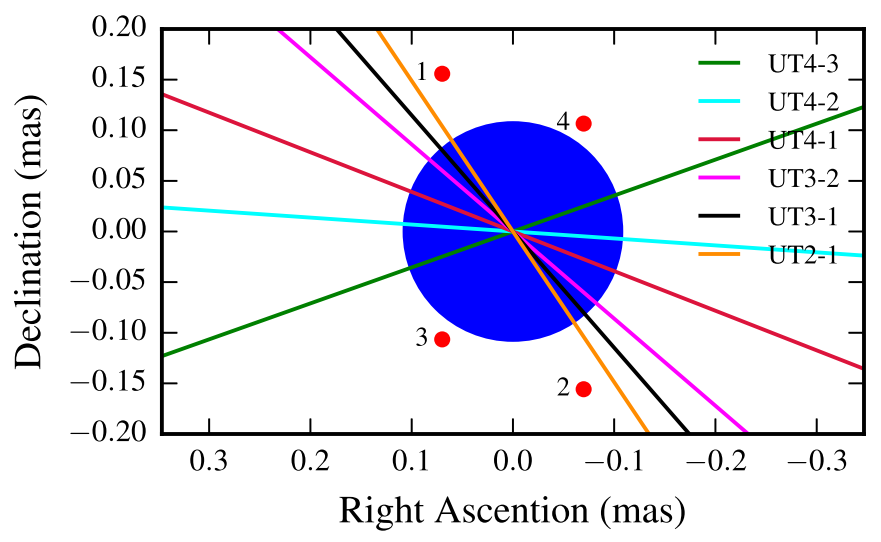

Figure 2. Baseline directions on the sky plane. Also shown are the donor star (photospheric radius $\sim 70 R_{\odot}$ ) and the four predicted possible positions of the pulsar (red) on the sky plane at the time of observation. For details, see Appendix A.

The baseline directions on the sky plane are shown in Figure 2, together with the predicted binary image at the time of observation. Because there is no astrometric information on the binary system, the exact position of the pulsar on the sky plane is not known. However, we show that we can narrow down its position to the four possibilities shown (see Appendix A).

\subsection{Data Reduction}

The data were reduced with the standard GRAVITY pipeline (version 0.9.6; Lapeyrere et al. 2014), which is based on the principle of the Pixel to Visibility Matrix (P2VM; Tatulli et al. 2007). An internal artificial light source is used to characterize the transition from pixel intensities to complex visibilities for each baseline in each of the two detectors (science and fringe tracker), which is then applied to the scientific observations (after appropriate sky subtraction). The wavelength calibration in this case (high resolution) is performed with an argon lamp. Absolute visibilities are calibrated by means of computing a transfer function using calibrator stars of known diameter, which is also used to calibrate the visibility and closure phases. The default values in the pipeline were used. In particular, we use the so-called VFACTOR to estimate the loss of coherence of the science channel using the fringe tracker data (which measures the phase deviations at a faster rate than the applied
Table 3

Interferometric Calibrators

\begin{tabular}{llcc}
\hline \hline Name & Spectral Type & Diameter (mas) & Reference \\
\hline HD 97550 & G8II/III & $0.828 \pm 0.008$ & (a) \\
HD 110532 & G8Ib/II & $0.804 \pm 0.008$ & (a) \\
\hline
\end{tabular}

Note.

a Mérand et al. (2005).

corrections), and rescale the science absolute visibilities accordingly.

The interferometric calibrators used are listed in Table 3. These stars were also used as telluric calibrators for the spectrum. As cool supergiants, they are expected to contain very weak absorption lines of hydrogen. In particular, by dividing by an approximate telluric spectrum, ${ }^{15}$ we checked that there was no remaining $\mathrm{Br} \gamma$ or He I line to be removed within the noise level of the spectrum. Unfortunately, the calibrator stars contain $\mathrm{CO}$ absorption bands in the red part of the spectrum, which is also affected by telluric lines. Therefore, we do not show wavelengths $\gtrsim 2.20 \mu \mathrm{m}$. This region shows neither discernible spectral lines nor interferometric signatures above the noise level.

The pipeline reports a wavelength calibration with absolute accuracy of $\sim 1$ spectral resolution element $\left(40 \mathrm{~km} \mathrm{~s}^{-1}\right)$. Since we can achieve statistical errors that are smaller than that when fitting strong emission lines, we cross-correlated (IRAF, XCSAO package) the uncorrected spectra with the model telluric spectrum in order to reduce the systematic uncertainty in the wavelength calibration. We found a global shift $\sim-60 \pm 5 \mathrm{~km} \mathrm{~s}^{-1}$ consistent for both calibrators and science spectra, and applied the correction.

\section{Spectroscopic Analysis}

\subsection{Results}

Currently, the most valid spectral classification of Wray 977 is an early blue hypergiant, $\mathrm{B} 1 \mathrm{Ia}+$, based on high-resolution optical spectra (Kaper et al. 2006). Figure 3 shows the $K$-band spectrum obtained with GRAVITY, and comparison spectra of $\zeta^{1}$ Sco, HD 169454 and HD190603, isolated stars of similar spectral type (Hanson et al. 1996). The most striking differences of Wray 977 are its stronger emission in He I $2.059 \mu \mathrm{m}$ and $\mathrm{Br} \gamma$ in emission rather than absorption. The spectrum in Figure 3 has been degraded to a worse resolution for a better comparison with the other stars. The spectrum at the original resolution is shown in Appendix C.

Table 4 shows the identified lines and their measured radial velocities from Gaussian fits (all wavelengths referred to are in vacuum). The He I absorption doublet lines were fit jointly with separate Gaussians for each line, and the He I emission line was fit jointly with a P-Cygni absorption component. The errors shown combine the statistical errors from the fit with the estimated $5 \mathrm{~km} \mathrm{~s}^{-1}$ error on the wavelength calibration. In practice, the error is dominated by systematic effects caused by the limited spectral resolution and imperfect telluric correction. The velocities were converted to the heliocentric frame.

\footnotetext{
15 Taken from ESO Spectroscopic Standards: http://www.eso.org/sci/ facilities/paranal/decommissioned/isaac/tools/spectroscopic_standards.html.
} 


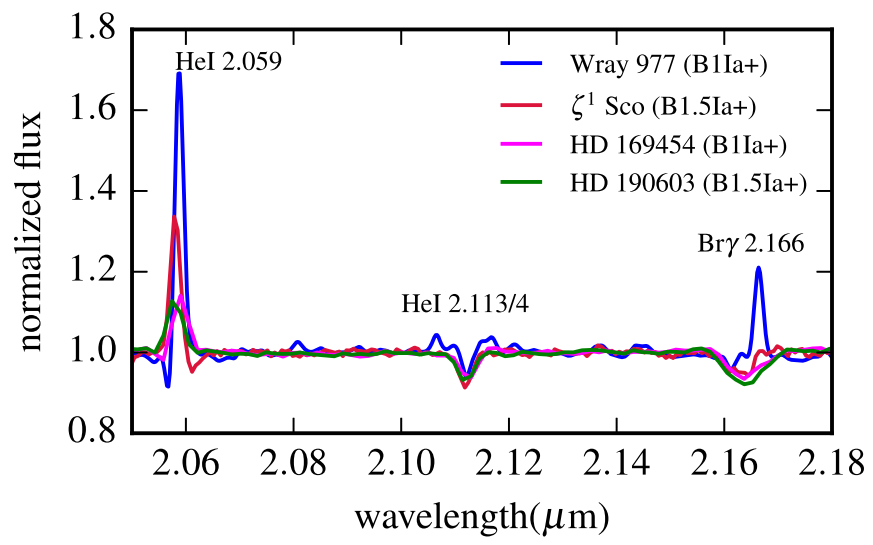

Figure 3. Comparison of Wray 977's GRAVITY spectrum with isolated stars of similar spectral type (Hanson et al. 1996). The GRAVITY spectrum has been degraded to the resolution of the $\zeta^{1}$ Sco spectrum $(R \sim 1500)$. The other two spectra have slightly lower resolution, $R \sim 800$. Note the more prominent He I $2.059 \mu \mathrm{m}$ emission and the $\mathrm{Br} \gamma$ line in emission for Wray 977. The stars have different wind properties, with Wray 977 having the densest wind.

Table 4

Spectral Lines Identified

\begin{tabular}{lc}
\hline \hline Line (Rest Wavelength in Vacuum) & Measured Velocity $\left(\mathrm{km} \mathrm{s}^{-1}\right)$ \\
\hline He I $2.0587 \mu \mathrm{m}$ & $+29 \pm 6 \mathrm{~km} \mathrm{~s}^{-1}$ \\
He I $2.1126 \mu \mathrm{m}$ & $-43 \pm 10 \mathrm{~km} \mathrm{~s}^{-1}$ \\
He I $2.1138 \mu \mathrm{m}$ & $+2 \pm 15 \mathrm{~km} \mathrm{~s}^{-1}$ \\
$\operatorname{Br} \gamma 2.16612 \mu \mathrm{m}$ & $+55 \pm 7 \mathrm{~km} \mathrm{~s}^{-1}$
\end{tabular}

The double He I $2.113 \mu \mathrm{m}, 2.114 \mu \mathrm{m}$ absorption lines do not show any interferometric signature relative to the continuum above the noise level, which could mean that they are photospheric. If this were true, they would trace the systemic velocity of the system as well as the radial velocity of the hypergiant (which is very small, $|v|<10 \mathrm{~km} \mathrm{~s}^{-1}$; Kaper et al. 2006). We obtain inconsistent results for the radial velocity of the two lines, which could be due to wind contamination. If that is the case, the lack of discernible interferometric signatures is not surprising as the lines are very weak.

\subsection{Discussion}

The He I $2.059 \mu \mathrm{m}$ line has an unsaturated P-Cygni profile, which suggests an optically thin wind. This line is highly sensitive to temperature and wind properties and becomes very active in $\mathrm{OB}$ supergiants, acting as a tracer of extended atmospheres (Hanson et al. 1996). Wray 977 has an estimated mass-loss rate $\sim 5-10 \times$ higher than the comparison stars shown, which is consistent with the stronger emission.

The $\mathrm{Br} \gamma$ in emission in Wray 977 is a clear deviation from the isolated comparison stars. One explanation could be that its denser wind drives the line into emission. Unfortunately, these are the only currently known galactic early-B hypergiants of subtype earlier than 2 (Clark et al. 2012), so this hypothesis cannot be tested observationally. Using detailed stellar atmosphere codes to test this hypothesis is beyond the scope of this paper. Preliminary results (F. Martins 2016, private communication) and previous work (Clark et al. 2003) suggest that this could indeed be the case.

Another possibility is that the $\mathrm{Br} \gamma$ emission could be caused by denser accretion structures present in the system. As a recombination line, $\mathrm{Br} \gamma$ emission is usually very sensitive to density (Kudritzki \& Puls 2000). There are many reports in the literature of $\mathrm{Br} \gamma$ emission lines in X-ray binary systems originating from the accretion disk and its wind. Shahbaz et al. (1999) report on a double-peaked $\operatorname{Br} \gamma$ emission line for the LMXB V616 Mon, in which the donor star is a K-type dwarf that should not show such an emission line. Bandyopadhyay et al. (1999) reports on $\mathrm{Br} \gamma$ lines with a P-Cygni shape from the LMXB systems Sco X-1 and GX13 + 1. In the latter, the donor star is a K-type giant that is not expected to have emission in $\operatorname{Br} \gamma$, whereas in the former the wind terminal velocity is too high to be associated with the O-type donor star wind. In both cases, an accretion disk wind is evoked to explain the emission. Perez \& Blundell (2009) report on a spectroscopic campaign to decompose the $\mathrm{Br} \gamma$ emission line of the HMXB and microquasar SS 433, and are able to find several emission components, including a double-peaked accretion disk component. Also in this case, the A-type donor star supergiant is not expected to show such an emission line. In several of these cases, He I lines in the $K$ band, most notably He I $2.059 \mu$ m, are also in emission.

In HMXBs such as BP Cru, where a stable accretion disk is not expected, associating $\mathrm{Br} \gamma$ or $\mathrm{He} \mathrm{I}$ line emission with an accretion structure is less obvious. However, this possibility should not be excluded in the case of BP Cru, since a gas stream of enhanced density that could be dominating the massloss rate is expected to be present.

\section{Interferometric Results}

Here we focus on the main results from the interferometric data. We divide this section into two parts: continuum visibilities and spectral differential visibilities. For the purposes of data analysis, the seven files were averaged, with the corresponding $(u, v)$ coordinates averaged linearly, as appropriate given the short time interval ( $\sim 1 \mathrm{hr} 20$ minutes) spanned by the files. Such an interval is also negligible compared to the orbital period and X-ray variability timescale.

\subsection{Continuum Size and Asymmetry}

Here we estimate an upper limit on the continuum size from the continuum visibility amplitudes. The most reliable visibility amplitude estimator is the squared visibility modulus of the fringe tracker (FT), since it measures the fringe visibility within the coherence time of the atmosphere. The FT operates at low resolution $(R \sim 20$, or five spectral channels across the $K$ band), which makes it useful for measuring continuum interferometric observables. As will be shown later, the emission lines are only marginally resolved, and therefore their effect on the visibilities of the FT spectral channels can be neglected.

We note that the continuum closure phases are zero to within the noise limit $\left(\mathrm{rms}<1^{\circ}\right)$ on all baselines. The closure phase is much more robust to systematic errors than the visibility amplitudes, and therefore there is strong indication for a symmetrical continuum emission. Since, in addition, the source is very close to unresolved, there is no big difference between using a disk, Gaussian, or any similar model for the continuum $|V|$. We choose a uniform disk model with the angular diameter as the only parameter.

Figure 4 shows the squared visibility modulus measured by the FT, averaged over the five spectral channels for each 


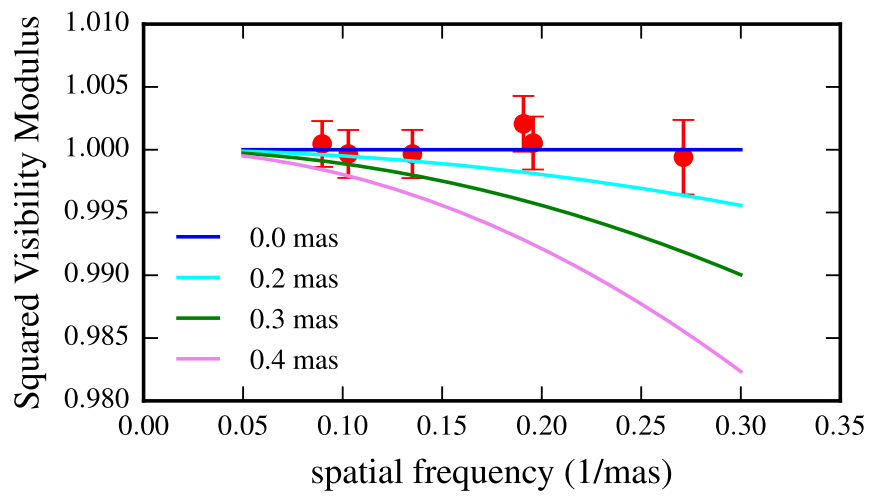

Figure 4. Continuum visibility amplitudes (spectrum average) measured by the fringe tracker. Disk models with varying angular diameters are shown for comparison.

baseline. The error bars include the measurement errors from the science object, as well as from the calibrator object and the calibrator diameter's systematic uncertainty $\sim 1 \%$. Disk models with the indicated angular diameters are also plotted for comparison.

The data are most consistent with an unresolved continuum of size $\theta_{d} \lesssim 0.2$ mas. Because the continuum size is in the very challenging limit that is well below the interferometer canonical resolution $\theta \ll \frac{\lambda}{|\boldsymbol{B}|} \sim 3$ mas, the measurements are very sensitive to systematic errors between baselines. We therefore restrain from a formal fit and restrict to providing a very conservative upper limit to the continuum size $\theta_{d} \lesssim 0.4$ mas. Structures larger than this are clearly inconsistent with the data, as shown in Figure 4.

\subsection{Differential Visibilities and Phases}

To treat the differential visibility signatures, we averaged the seven files after normalizing the visibility amplitudes to an unresolved continuum. The visibility phases are output from the pipeline already mean and slope subtracted i.e., as differential quantities.

Figure 5 shows the differential visibility amplitudes across the $\mathrm{Br} \gamma$ line for the six baselines at hand. The photosphericcorrected flux ratio (see Appendix B) between the continuum and the line emission is also shown for comparison. In general, the visibility amplitudes show, for some baselines, a decrease at the lines relative to the continuum, which is indicative of extended or multicomponent emission. However, the peak of the $|V|$ drop does not happen at the center of the line, but rather it is displaced to the blue side. Figure 6 shows the differential visibility phases. They show larger, negative values on the blue side of the line and, for some baselines, smaller, positive values on the red side of the line. Such "S-shaped" differential visibility signatures across a line are typical interferometric tracers of rotation e.g., they are often observed in Be stars, in which they are attributed to extended equatorial disks, but in these systems the blue and red phase signatures are roughly symmetric (Meilland et al. 2012). The black lines in the plots are model-independent fits to the data and will be discussed in the following section.

Appendix $\mathrm{C}$ shows the differential visibilities across the spectrum. Interferometric features similar to $\mathrm{Br} \gamma$ in both differential visibility amplitudes and phases are also found across the He I $2.059 \mu \mathrm{m}$ emission line. However, this region of the spectrum suffers from a particularly high level of noise due to the GRAVITY metrology laser and the large telluric absorption. For instance, the rms in the visibility amplitude, estimated from the scatter in the continuum region around the lines, is $0.4 \%$ and $1.2 \%$ for $\mathrm{Br} \gamma$ and $\mathrm{He} \mathrm{I}$, respectively. Similarly, the corresponding values for differential visibility phases are 0.2 and 0.6 . That, in addition to the more complicated (P-Cygni) shape of the line, led us to focus our analysis on the $\operatorname{Br} \gamma$ line. We show in Figure 7 the visibility signatures across the $\mathrm{He}$ I $2.059 \mu \mathrm{m}$ line for some representative baselines.

Several factors point to the credibility of such features. The wavelength alignment between the extracted spectrum for each telescope agrees to $<\frac{1}{2}$ of a resolution element. Similar features are not found at other lines in the spectrum, either related to the science object (e.g., He I 2.113/4 $\mu \mathrm{m}$ ) or telluric. Moreover, they show up with different strengths for different baselines (as expected for any reasonable interferometric model) and are consistent between the two emission lines. Finally, for the differential visibility amplitudes, the features are strongest in the three baselines that encompass all of the four telescopes, whereas for the differential visibility phases a signature is detectable in five of the six baselines.

\subsection{Closure Phases}

Closure phases are sums of visibility phases formed in a closed triangle of baselines that are independent of telescope errors. For this reason, they are robust probes of asymmetry. As mentioned above, the closure phases across the continuum are zero to within the noise on all four baseline triangles (only three are independent). In theory, differential closure phases are not independent measurements from what has already been presented since they are derived from linear combinations of differential phases.

Appendix C (and Figure 8 for a closer look at the $\mathrm{Br} \gamma$ line) shows that the differential closure phases across the emission lines vanish to within the noise level. Even though the differential closure phases are naturally noisier than the individual baseline differential visibility phases by $\approx \sqrt{3}$ (rms $=0.4)$, the fact that they vanish might be puzzling at first since the differential visibility phases are nonzero and therefore indicate the presence of asymmetry. This will be clarified in the following section.

\section{Discussion}

\subsection{Continuum}

The photospheric radius $R\left(\tau_{\text {Ross }}=2 / 3\right)=70 R_{\odot}$ and the distance of $3 \mathrm{kpc}$ to Wray 977 (Kaper et al. 2006) imply a photosphere angular diameter $\theta \approx 0.2$ mas. Our continuum size measurements are therefore consistent with a size $\lesssim 2 \times$ the photosphere diameter, using our conservative upper limit referenced above. For hot stars with strong winds, the observed continuum emission in the infrared is a combination of blackbody thermal emission around the photosphere region as well as bound-free and free-free emission in the optically thin wind. Kaper et al. (2006) compare the SED of Wray 977 with a Kurucz model with the same temperature and find a strong infrared excess, associated with emission from the wind. However, at the maximum wavelength probed by GRAVITY, $\sim 2.5 \mu \mathrm{m}$, the wind contribution is still relatively small, $\sim 20 \%$ of the flux. Therefore, it is expected that the continuum in the $K$ band is still dominated by the photosphere rather than the 

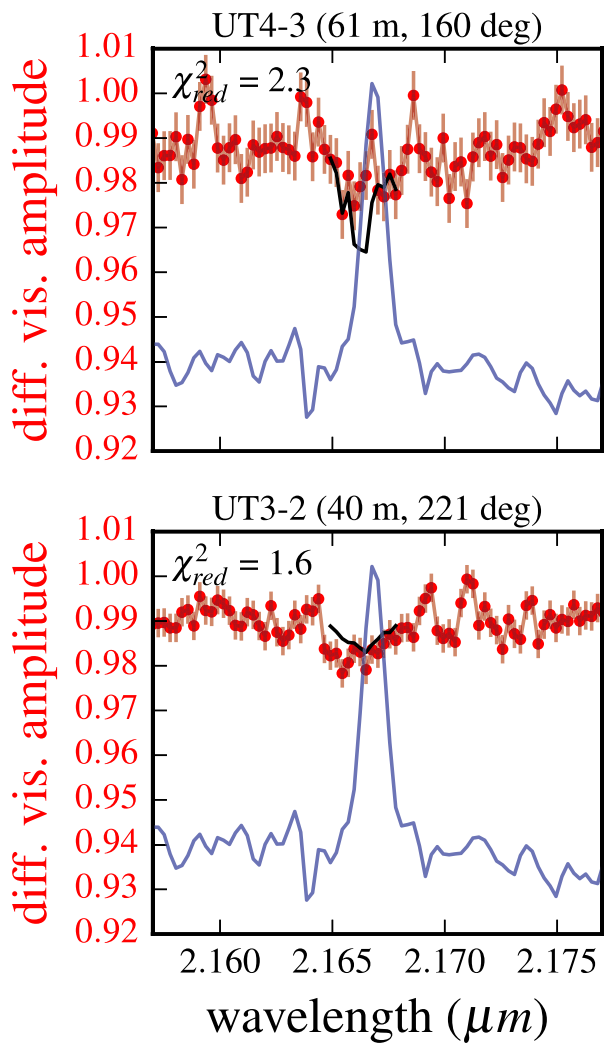

UT4-2 (88 m, $184 \mathrm{deg})$

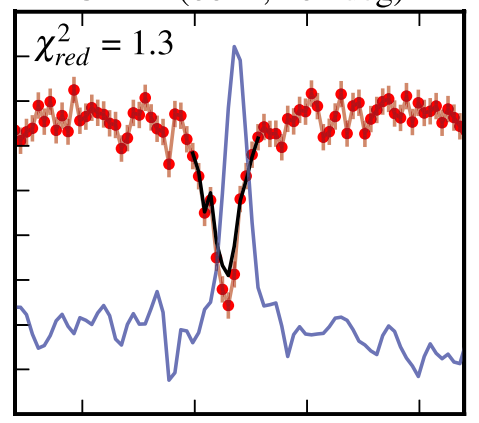

UT3-1 (86 m, $229 \mathrm{deg})$

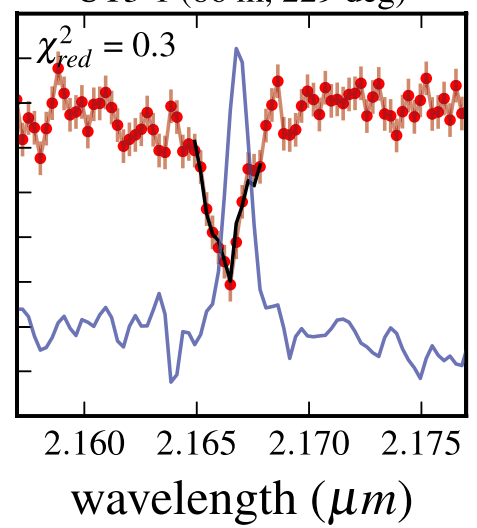

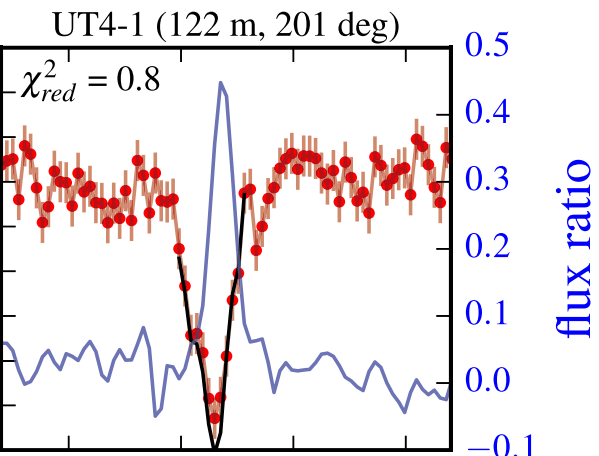

UT2-1 (46 m, $236 \mathrm{deg}$ )

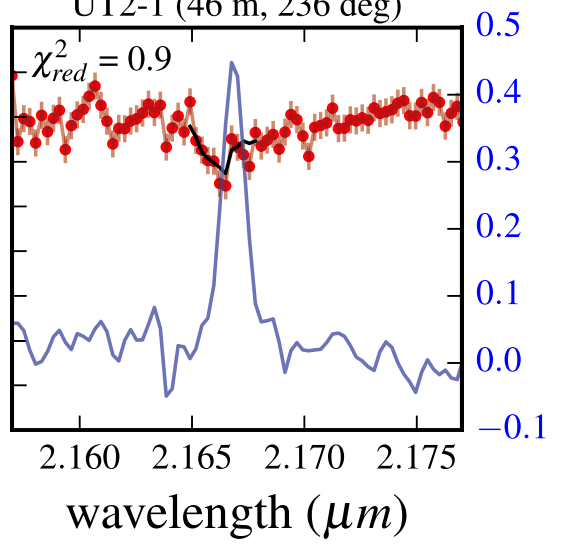

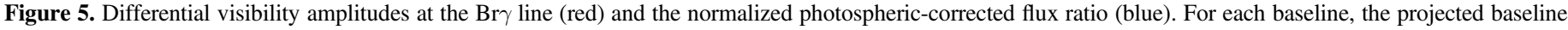
length and the position angle are also shown. In black, we show model-independent fits to the visibility amplitudes (see text for details).

wind. This is consistent with the interferometric results presented here. Furthermore, the lack of a resolved structure in the near-infrared continuum also argues against the presence of a circumstellar disk, which is often seen in Be stars as extended continuum emission in the $K$ band with FWHM $\gtrsim 2 D_{*}$ (Meilland et al. 2012).

\subsection{Differential Visibilities}

The main advantage of using spectral differential visibility measurements is that they are much less susceptible to systematic errors that can affect the absolute visibility quantities. The errors in fringe contrast and phase are, in general, monotonic functions of the phase difference caused by spurious OPDs between baselines, $\Delta \phi=\frac{2 \pi}{\lambda} \mathrm{OPD}$. The error in the differential quantities will then have the form $f(d \Delta \Phi) \approx f\left(-2 \pi \frac{\text { OPD }}{\lambda} \frac{d \lambda}{\lambda}\right)$, which is greatly reduced with respect to the non-differential error when $\frac{d \lambda}{\lambda} \ll 1$, which is the case, for example, when using the wavelength of a narrow line compared to the continuum around it. On top of that, the differential quantities are not affected by wavelength-independent errors and are robust to low-order spurious effects along the spectrum given the narrowness of the spectral lines.

\subsubsection{Model-independent Analysis in the Marginally Resolved Limit}

The downside of spectral differential quantities is that, when imaging is not possible, their ultimate interpretation relies on knowing the spectral decomposition of the line, in case there is more than one emission component. Given the likely complex nature of the source in question and the many possible components in the system (hypergiant photosphere, wind, pulsar, gas stream, accretion disk, etc.), it would be useful to derive model-independent properties of the image that any model would have to reproduce. In general, this is not possible without image reconstruction, which requires a much denser $u-v$ sampling than what we have available here.

However, when the interferometric signatures are small, such as is the case here, spectral differential quantities nicely fit into the special framework of the marginally resolved limit in interferometry. Lachaume (2003) lays out the formalism of this limit, showing that the visibility signals can be related to the moments of the flux distribution in a model-independent way. This technique has been applied extensively in the interpretation of spectral differential visibility phases as photocenter displacements (Monnier \& Allen 2013). Because here we also want to use the visibility amplitudes to estimate the secondorder moments, we review the basic idea of the method and extend it to spectral differential visibilities.

From the Van Cittert-Zernike theorem,

$$
F(\boldsymbol{u})=\iint I(\boldsymbol{\sigma}) e^{-2 \pi i \boldsymbol{\sigma} \cdot \boldsymbol{u}} d l d m
$$

where $F$ is the coherent flux, $I$ is the source intensity distribution, $\boldsymbol{\sigma}=(l, m)$ are the object coordinates on sky, and $\boldsymbol{u}=$ $\frac{\boldsymbol{B}}{\lambda}=(u, v)$ is the baseline vector. In the following, it will be useful to define the moments of the intensity distribution about the origin as

$$
\mu_{p q}=\iint I(\sigma) l^{p} m^{q} d l d m
$$

so that, for example, the zero-order moment $\mu_{00}$ is the total intensity and the normalized first-order moments $l_{1}=\frac{\mu_{10}}{\mu_{00}}$ and 

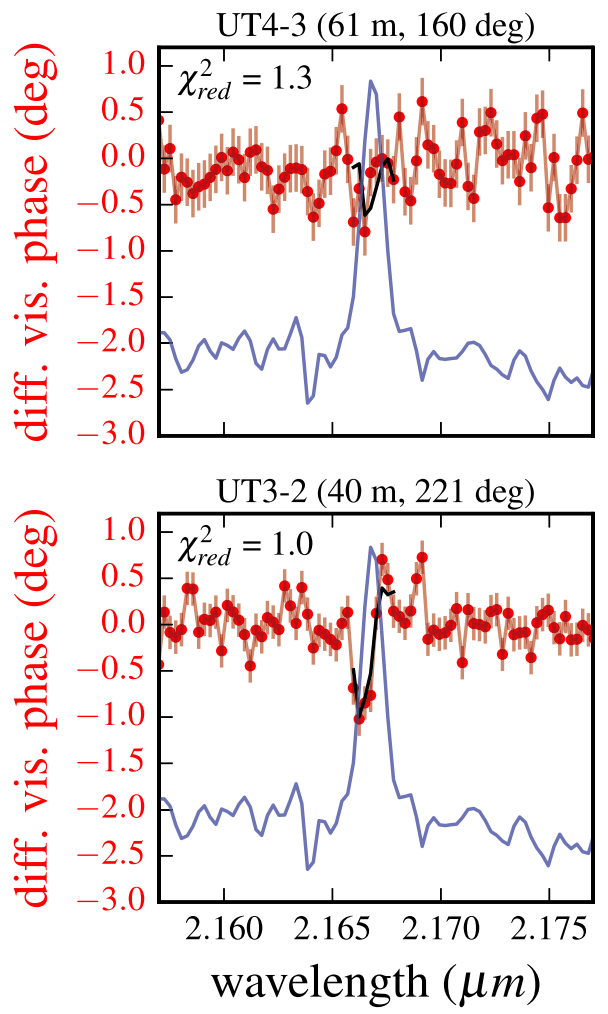
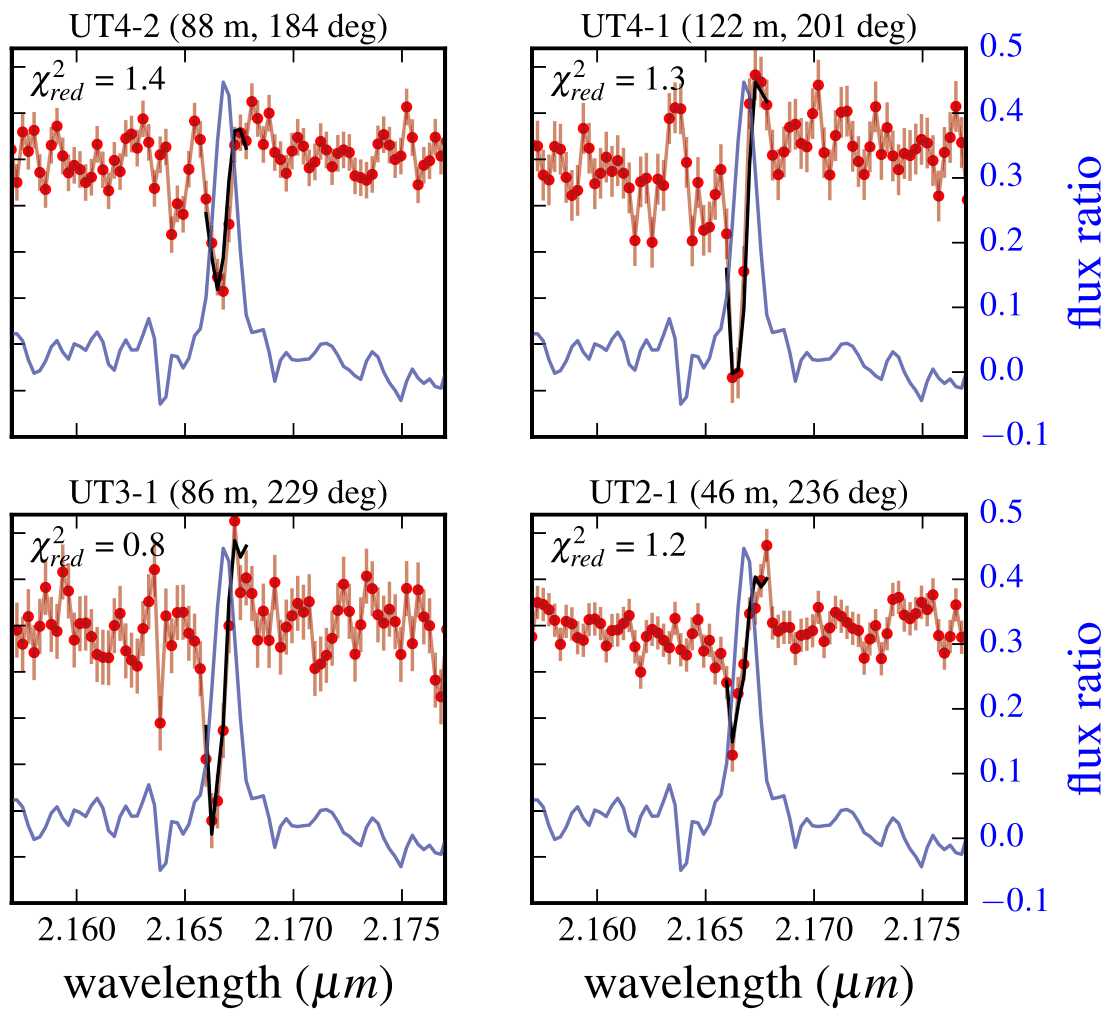

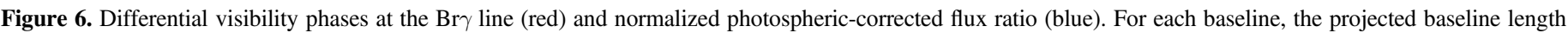
and the position angle are also shown. In black, we show model-independent fits to the visibility phases (see text for details).
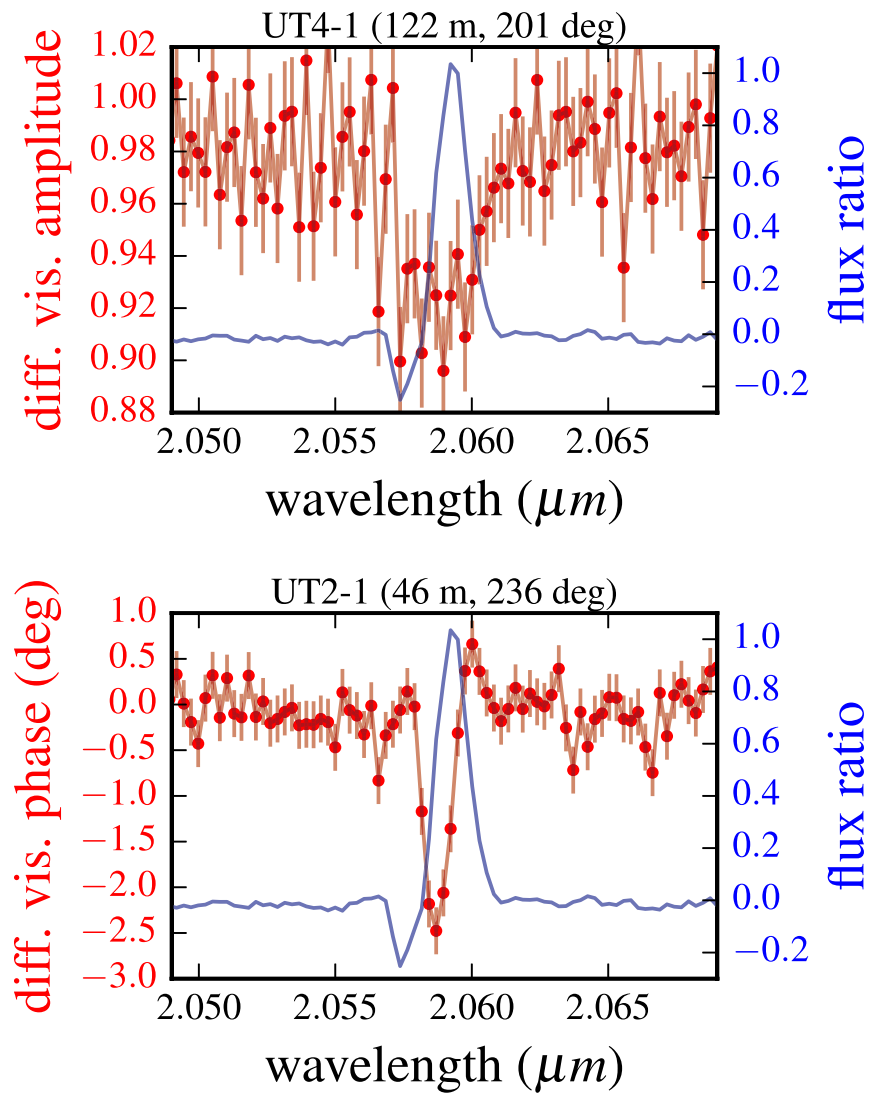

Figure 7. Differential visibility amplitudes and phases across the He I $2.059 \mu \mathrm{m}$ line for some representative baselines. The features agree with those seen in $\mathrm{Br} \gamma$, but are, in general, noisier due to instrumental and atmospheric effects. $m_{1}=\frac{\mu_{01}}{\mu_{00}}$ are the centroid positions along the $l$ - and $m$-axes, respectively. We can expand the complex exponential term in the integral of Equation (1) in a Taylor series:

$$
\begin{aligned}
e^{-2 \pi i \boldsymbol{\sigma} \cdot \boldsymbol{u}}= & 1-2 \pi i(\boldsymbol{\sigma} \cdot \boldsymbol{u})-2 \pi^{2}(\boldsymbol{\sigma} \cdot \boldsymbol{u})^{2} \\
& +\frac{4 \pi^{3} i}{3}(\boldsymbol{\sigma} \cdot \boldsymbol{u})^{3}+\mathcal{O}\left((\boldsymbol{\sigma} \cdot \boldsymbol{u})^{4}\right),
\end{aligned}
$$

which allows the use of approximations when

$$
|\boldsymbol{\sigma} \cdot \boldsymbol{u}| \ll 1 \Leftarrow|\boldsymbol{\sigma}| \ll \frac{\lambda}{|\boldsymbol{B}|},
$$

i.e., when the source is sufficiently unresolved for a given baseline vector. Using the standard definition of the complex visibility,

$$
V(\boldsymbol{u})=\frac{F(\boldsymbol{u})}{F(0)}=\frac{F(\boldsymbol{u})}{\mu_{00}},
$$

it follows that

$$
V(\boldsymbol{u}) \approx 1-2 \pi i w_{1}-2 \pi^{2} w_{2}+\frac{4 \pi^{3} i}{3} w_{3},
$$

where

$$
w_{i}=\frac{1}{\mu_{00}} \int I(\boldsymbol{\sigma})(\boldsymbol{\sigma} \cdot \boldsymbol{u})^{i} d l d m,
$$

To first order in $\boldsymbol{\sigma} \cdot \boldsymbol{u}$, the phase of the visibility is

$$
\arg (V(\boldsymbol{u})) \approx \arctan \left(\frac{-2 \pi w_{1}}{1}\right) \approx-2 \pi w_{1},
$$



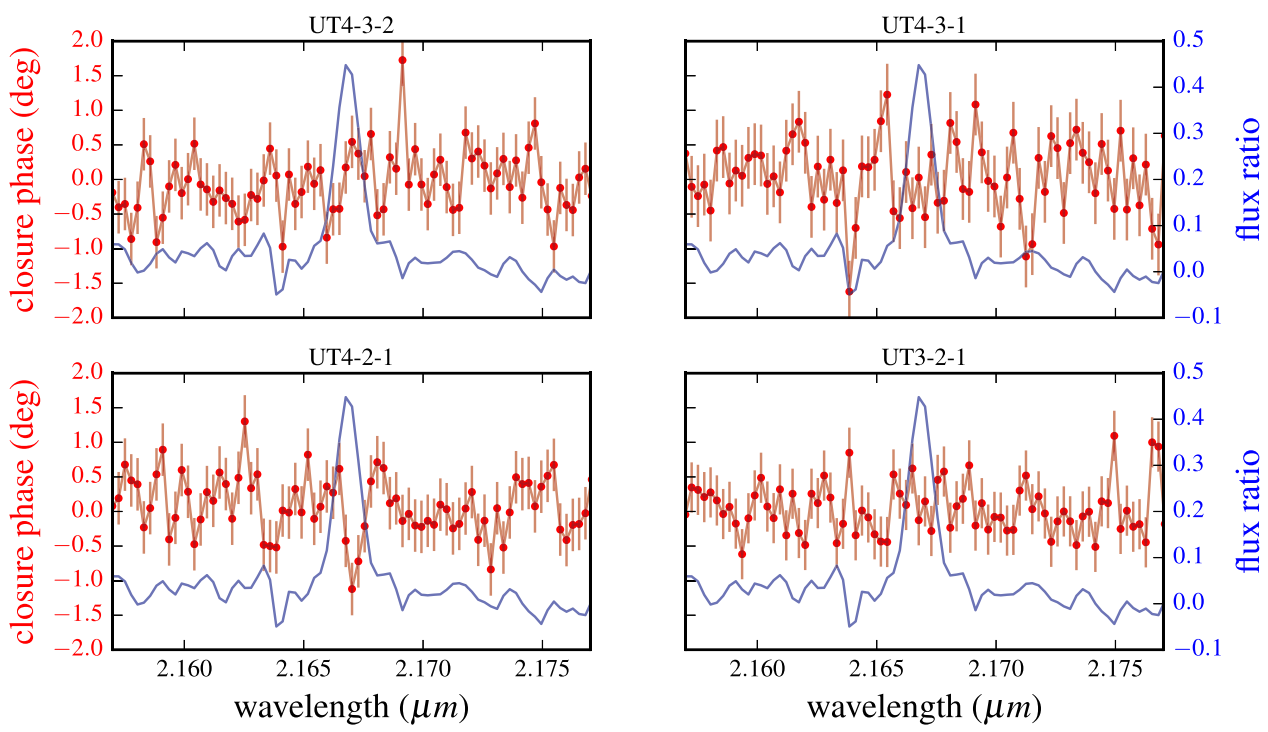

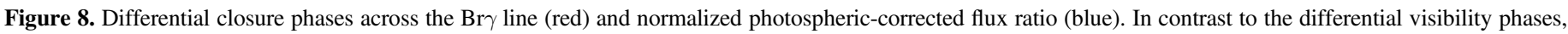
there is no clearly distinguishable feature within the noise.

since $w_{1} \ll 1$. Calling $\boldsymbol{x}=\left(l_{1}, m_{1}\right)$ the centroid positions for the given intensity distribution,

$$
\arg (V(\boldsymbol{u})) \approx-2 \pi \boldsymbol{u} \cdot \boldsymbol{x} .
$$

For two given images $a$ and $b$ at the same spatial frequency $\boldsymbol{u}$, the differential phase

$$
\begin{aligned}
\Delta \phi_{b a} & =\arg \left(V_{b}\right)-\arg \left(V_{a}\right) \approx-2 \pi\left(w_{1, b}-w_{1, a}\right) \\
& \approx-2 \pi \boldsymbol{u} \cdot\left(\boldsymbol{x}_{b}-\boldsymbol{x}_{a}\right) .
\end{aligned}
$$

Equation (10) shows that differential visibility phases give model-independent centroid displacements along the baseline direction for close to unresolved sources. If two or more baselines are available, this allows the centroid displacement $\Delta \boldsymbol{x}_{\mathrm{ab}}$ to be solved or fit. Note that the differential phase is proportional to the baseline length. Therefore, for this approximation method to work in practice as a robust, model-independent estimation, we must have sufficiently small differential phase errors so that a signal can be measured even with a small enough baseline so that the sources remain very close to unresolved. Fortunately, this is exactly the case in spectral differential phase measurements, for which the error is much smaller than the absolute phase errors plagued by systematics.

We can go one order further by using differential visibility amplitudes. To second order in $\boldsymbol{\sigma} \cdot \boldsymbol{u}$,

$$
\begin{gathered}
|V(\boldsymbol{u})| \approx\left(\left(1-2 \pi^{2} w_{2}\right)^{2}+\left(2 \pi w_{1}\right)^{2}\right)^{1 / 2} \\
\approx 1+2 \pi^{2} w_{1}^{2}-2 \pi^{2} w_{2}+2 \pi^{4} w_{2}^{2} \\
\approx 1+2 \pi^{2}\left(w_{1}^{2}-w_{2}\right),
\end{gathered}
$$

since $w_{1}, w_{2} \ll 1$ and we must expand to second order since the first-order term alone would result in $|V|>1$. Note that in this expression the visibility amplitude depends on $w_{1}$, i.e., on the centroid of the image and therefore on the absolute phase, which is not available from single-axis interferometry. Even the differential visibility amplitude between the two images $a$ and $b$ with this expression would depend on $w_{1, b}^{2}-w_{1, a}^{2}$, whereas only $w_{1, b}-w_{1, a}$ is available from the differential visibility phase as shown above. In order to circumvent this, it is useful to define the moments of the image with respect to the centroid $\boldsymbol{x}=\left(l_{1}, m_{1}\right)$,

$$
\tilde{\mu}_{p q}=\iint I(\sigma)\left(l-l_{1}\right)^{p}\left(m-m_{1}\right)^{q} d l d m,
$$

so that, for example, the normalized second-order moments $\tilde{l}_{2}=\frac{\tilde{\mu}_{20}}{\mu_{00}}$ and $\tilde{m}_{2}=\frac{\tilde{\mu}_{02}}{\mu_{00}}$ are the variances about the centroid position along the $l$ - and $m$-axes, respectively, and $\frac{\tilde{\mu}_{11}}{\mu_{00}}$ is the covariance. Analogously, we define

$$
\tilde{w}_{i}=\frac{1}{\mu_{00}} \int I(\boldsymbol{\sigma})((\boldsymbol{\sigma}-\boldsymbol{x}) \cdot \boldsymbol{u})^{i} d l d m
$$

It is straightforward to show directly from the definitions that $\tilde{w}_{2}=w_{2}-w_{1}^{2}$, so that

$$
|V| \approx 1-2 \pi^{2} \tilde{w}_{2}
$$

where from Equation (15), for a given baseline $\boldsymbol{u}=(u, v)$,

$$
\tilde{w}_{2}=u^{2} \frac{\tilde{\mu}_{20}}{\mu_{00}}+v^{2} \frac{\tilde{\mu}_{02}}{\mu_{00}}+2 u v \frac{\tilde{\mu}_{11}}{\mu_{00}} .
$$

Note that this is a better definition since these moments are about the image centroid rather than an arbitrary phase center. Given two images $a$ and $b$, for example at the continuum and at a spectral line, the differential visibility amplitude is therefore

$$
\Delta|V|_{b a}=|V|_{b}-|V|_{a} \approx-2 \pi^{2}\left(\tilde{w}_{2, b}-\tilde{w}_{2, a}\right) .
$$

If three or more baselines are available, it is possible to solve for the difference in variances and covariance about the centroid between the continuum and the spectral line images. If a model for the continuum is available, differential visibility amplitudes allow robust estimates of the size and asymmetry of the image in the spectral line to be obtained.

Note that Equation (9) implies that, for any baseline triangle $\boldsymbol{u}_{1}+\boldsymbol{u}_{2}+\boldsymbol{u}_{3}=0$, the closure phase

$$
\arg \left(V\left(\boldsymbol{u}_{1}\right)\right)+\arg \left(V\left(\boldsymbol{u}_{2}\right)\right)+\arg \left(V\left(\boldsymbol{u}_{3}\right)\right)
$$




$$
=-2 \pi\left(u_{1}+u_{2}+u_{3}\right) \cdot x=0
$$

Therefore, the marginally resolved limit must be compatible with very small closure phases for all baselines. Lachaume (2003) shows that the closure phases are related to the third-order moments of the image distribution and are therefore expected to be much smaller than the visibility phases themselves in the marginally resolved limit.

The marginally resolved limit is applicable when $|\boldsymbol{\sigma} \cdot \boldsymbol{u}| \ll 1$. The translation of this condition into a minimum $|V|$ and the error incurred in the approximation are baseline and model dependent. Lachaume (2003) compared the exact versus the approximated visibilities for different simple models (binary, ring, Gaussian disk) and found that the approximation holds up to $|V| \gtrsim 0.9$ (see their Figure 4).

The validity of the marginally resolved limit for our data set is supported by the large visibility amplitudes $|V|>90 \%$, small $\left(<3^{\circ}\right)$ differential visibility phases, and vanishing closure phases (or closure phases that are much smaller than the individual visibility phases). Using the above formalism, we fit for the difference in centroid between the image at the continuum and the image at the spectral line (which includes emission from both the continuum and the line) using the differential visibility phases of the six baselines per spectral channel (therefore, there are six measurements and two parameters). The best-fit model (and corresponding $\chi_{\text {red }}^{2}$ ) is shown in Figure 6 (black line). For this and all subsequent model fits, we use a Markov Chain Monte Carlo technique as implemented in the publicly available EMCEE code (ForemanMackey et al. 2013) using uniform priors. We only fit the spectral channels in which there is emission line flux above the continuum noise level.

The consistency between the six baselines is further confirmation that the marginally resolved limit is valid. The resulting centroids on the sky plane for each wavelength across the emission line are shown in Figure 9. The error bars shown correspond to the $16 \%$ and $84 \%$ marginalized quantiles. The red part of the line must have a smaller ( $\sim 10 \mu$ as $)$ centroid shift with respect to the continuum image than the blue part of the line ( $\sim 30 \mu$ as). This statement is model independent. Because the image at the line contains both a line as well as a continuum contribution, we can estimate the barycenter of the line emission with respect to the continuum $($ at $(0,0))$ by scaling the model-independent centroids by $\frac{1+f}{f}$, where $f$ is the flux ratio between the continuum and line emission (see Appendix B). This, however, must be interpreted carefully since the line emission could have more than one component. The result is also shown in Figure 9. The resulting centroid positions suggest line emission offset from the continuum by less than the size of the binary orbit, with a spatial gradient across wavelengths and the bluest channels consistent with one of the possible positions of the pulsar on the sky plane.

Analogously, we fit for the difference in the second-order moments (variance and covariance) between the image in the continuum and the image along the emission line. In this case, there are six measurements and three parameters. The results are shown in Figure 5 (black line). Again, a consistency between baselines confirms the validity of the marginally resolved limit. The resulting variance difference in both R.A. and decl. as a function of wavelength is shown in Figure 10. Clearly, a higher variance is required on the blue side of the
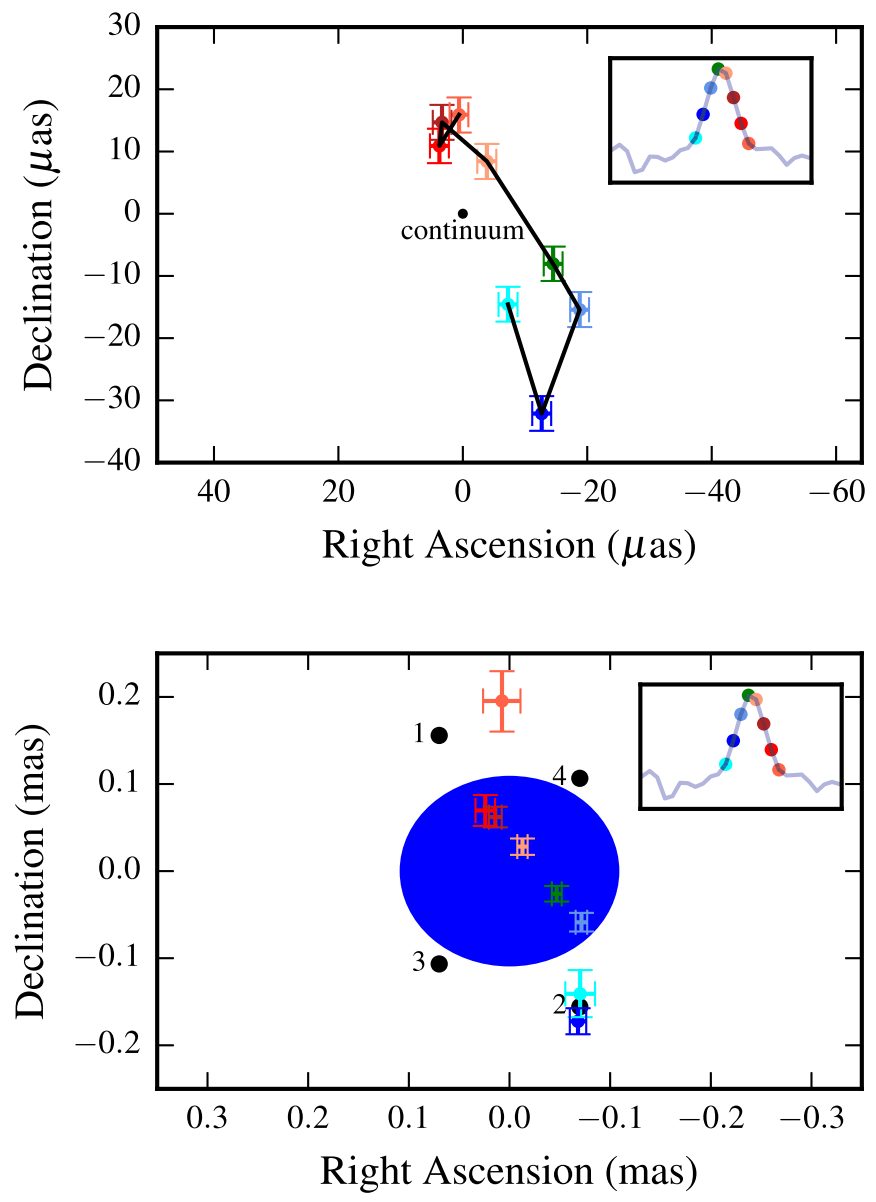

Figure 9. Top: model-independent centroid positions for each wavelength across the $\operatorname{Br} \gamma$ line (continuum is at $(0,0)$ ). The image on the blue side of the line has a larger centroid shift as compared to the image on the red side. Bottom: same as above, but using the flux ratio to derive the barycenter of the line emission. The hypergiant and the four predicted possible pulsar positions are also shown.

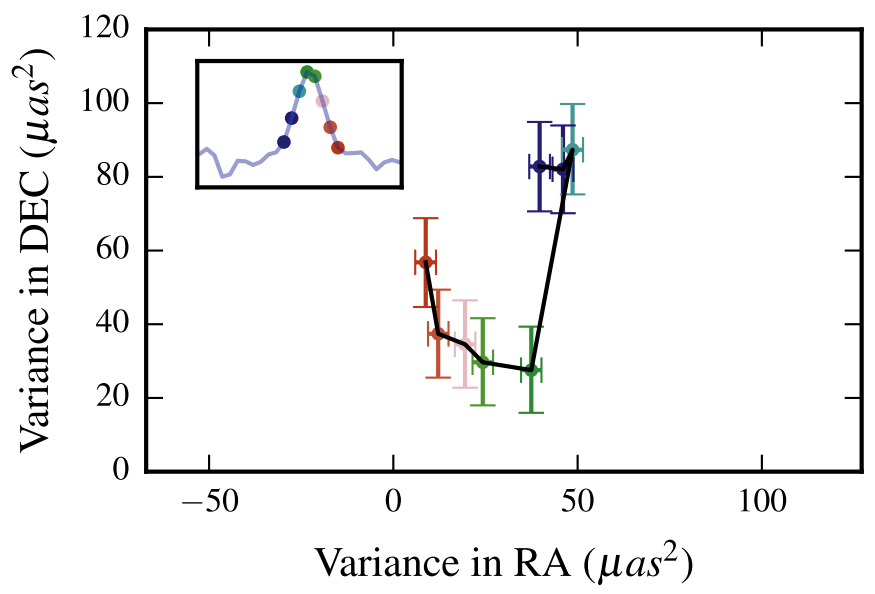

Figure 10. Model-independent variances of the image as a function of wavelength across the $\mathrm{Br} \gamma$ line. The blue part of the line has higher values, which suggests that the emission must be coming from larger scales.

line, implying that this part of the emission must come from larger scales. Also, the fact that the variances are not symmetrical in R.A. and decl. suggests an asymmetric emission structure. 


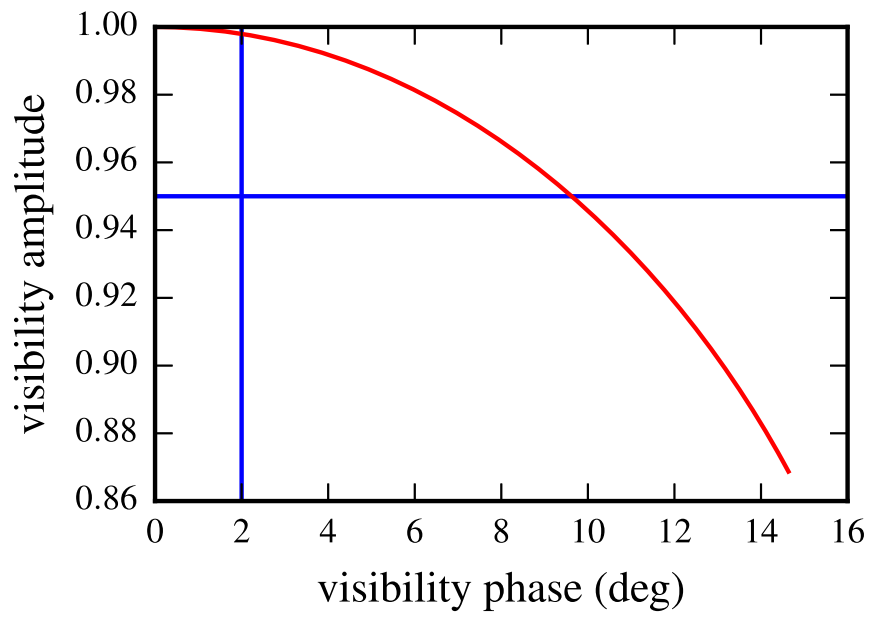

Figure 11. Visibility amplitude vs. phase as the separation is changed for a 1D binary model with flux ratio $f=0.3$ and $u$ coordinate $0.2 \mathrm{mas}^{-1}$. The measured visibility amplitudes $\sim 95 \%$ and phases $\sim 1^{\circ}-2^{\circ}$ are not compatible with this simple model.

The differential amplitude signatures are larger than expected from the differential phases. For example, for a binary model with compact components and flux ratio given by the spectrum, the binary separation as implied by the differential phases is one order of magnitude smaller than the one that would be necessary to produce the differential visibility amplitudes. This is illustrated in Figure 11, where we plot the visibility amplitude versus phase for a $1 \mathrm{D}$ binary model as the binary separation is changed. We choose a flux ratio $f=0.3$ and a $u$ coordinate $0.2 \mathrm{mas}^{-1}$, which are representative of our data. We can clearly see that visibility amplitudes $\sim 95 \%$ are not compatible with visibility phases $\sim 1^{\circ}-2^{\circ}$. This statement is robust and not dependent on the chosen $f$ and $u$.

\subsubsection{Simple Geometric Models}

The model-independent analysis in the context of the marginally resolved limit presented above allows the properties that any interferometric model has to satisfy in order to explain the data to be derived. In summary:

1. the image centroid must have a spatial gradient across the spectrum, with larger centroid deviations from the continuum at the blue side of the line, and in the opposite direction at the red side;

2. the image variance must also show such a gradient, with larger spatial extension also at the blue side of the line; and

3. small centroid displacements must coexist with largescale structure.

Fitting the data with complex hydrodynamic models which produce $\mathrm{Br} \gamma$ emissivity maps is beyond the scope of this paper. Instead, we restrict ourselves to the use of physically motivated, geometric models. We note that any interferometric model must deal with flux ratios, which are often degenerate with the spatial parameters. Whereas the simplest assumption is to use the spectrum to set the flux ratio, this only works if there is only one emission component. Since determining a complex spectral decomposition from interferometric data at moderate resolution is not possible, we limit ourselves to the simplest assumptions in the following models.

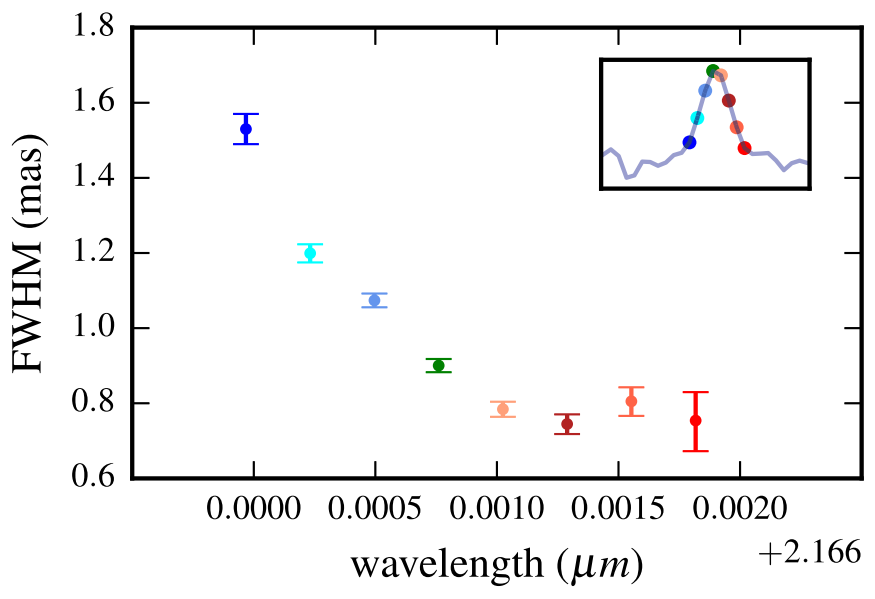

Figure 12. Wind size (FWHM) as a function of wavelength for a model in which the $\mathrm{Br} \gamma$ emission is dominated by the wind. Such a model predicts that there is still substantial wind emission at $4-7 \times R_{*}$, and that the blue (approaching) part of the wind is up to $\sim 2 \times$ more extended than the red (receding) part.

\subsubsection{Model A: Extended and Distorted Wind}

In this model, we assume that the $\mathrm{Br} \gamma$ emission is completely dominated by the hypergiant stellar wind. A spherically symmetric wind centered on the star would not be able to produce differential visibility phases with respect to the continuum; therefore, we allow the wind, which is modeled as a Gaussian, to be displaced from the center. For each wavelength channel across the $\operatorname{Br} \gamma$, we therefore model the complex visibility as

$$
V(\boldsymbol{u})=\frac{V_{\text {cont }}(\boldsymbol{u})+f e^{-\pi^{2}|\boldsymbol{u}|^{2} \frac{\theta_{d}^{2}}{4 \log 2}} e^{-2 \pi i \sigma_{0} \cdot \boldsymbol{u}}}{1+f},
$$

where $V_{\text {cont }}(\boldsymbol{u})$ is the continuum visibility, $f$ is the photosphericcorrected flux ratio between wind emission and continuum set by the spectrum, and the fit parameters are $\theta_{d}$, the FWHM of the wind, and $\sigma_{0}$, the centroid position of the wind.

This model is fit to both visibility amplitudes $\left(\chi_{\text {red }}^{2}=2.67\right)$ and differential visibility phases $\left(\chi_{\text {red }}^{2}=1.36\right)$. Because the (differential) closure phases can be derived from the visibility phases, they are not included in the fit; in other words, a good fit with respect to differential visibility phases should automatically be consistent with differential closure phases. The resulting centroid fits are identical to those shown in Figure 9 (bottom), as they should, since we are likewise assuming here that only one (spherically symmetric) structure contributes to the emission. The resulting wind sizes, as a function of wavelength, are shown in Figure 12.

The resulting wind FWHM (from $\sim 0.8$ mas on the red part of the wind up to $\sim 1.5$ mas on the blue part) would imply that there is substantial emission in $\operatorname{Br} \gamma$ up to $\sim 4-7 \times R_{*}$. On the other hand, the non-Lyman $\mathrm{H}$ lines in hot stars are usually recombination lines, which means that their source function is roughly Planckian and stays approximately constant throughout a wind that is at radiative equilibrium. At the same time, their opacity $\kappa \propto \rho^{2}$ is a very sensitive function of density, and for an accelerating wind with a fast-decaying density profile ( $\left.\rho \propto \frac{1}{r^{2} v(r)}\right)$, only the innermost $\left(\sim 1-1.5 R_{*}\right)$ regions of the wind would have a substantial contribution to the emission 
(Kudritzki \& Puls 2000). A varying temperature profile and the dependence of optical depth with velocity gradient $\left(\tau \propto \frac{d v}{d r}\right)$ might smooth the density decay, but it is unlikely to resolve the discrepancy in the case of Wray 977, where the CAK wind law (Castor et al. 1975) predicts a density at $4 R_{*}$ that is already $\sim \frac{1}{1000}$ of the value at $R_{*}$. A radiative transfer calculation to determine the emission region of $\mathrm{Br} \gamma$ in the wind is beyond the scope of this work; nonetheless, preliminary results (F. Martins 2016, private communication) show that a dense wind could indeed bring $\mathrm{Br} \gamma$ into emission, but the emission region would be sharply peaked between $\sim 1.3-3 R_{*}$, and therefore unable to account for such extended emission. Mid-infrared observations of BP Cru have detected the presence of dust and the possibility that the binary system is enshrouded by a disk-like circumstellar envelope $\sim 2$ mas (Servillat et al. 2014). Even though (i) the optical spectrum shows no evidence for a circumstellar disk, (ii) the interferometric signatures are not typical of a symmetric disk, and (iii) the near-infrared continuum is unresolved, there could be a connection between the very extended wind emission seen in these data and the reported dusty CS structure in the mid-infrared.

Another feature of the wind model is that the blue (approaching) side of the wind would have to be $\sim 1.5-2 \times$ more extended than the red (receding) part, where the pulsar is predicted to be at the time of the observation. This could be due to the X-ray illumination of the red part of the wind that hinders the radiative acceleration of the wind by photoionization.

The centroid shifts of the wind with respect to the continuum, necessary to explain the differential visibility phases, are small with respect to the size of the wind, $\left|\boldsymbol{\sigma}_{0}\right| / \theta_{d} \sim 10 \%$. Because a Gaussian image has no intrinsic phase, the small centroid shifts in the model might be indicative of an asymmetric wind structure. Such asymmetries could arise from a clumpy wind, or, more generally, from density fluctuations in the wind, which could be caused by the influence of the gravitational or radiation fields of the compact object. Although Kaper et al. (2006) found no evidence for wind clumping in Wray 977 from optical spectrum modeling, $\mathrm{X}$-ray light curves and column density measurements often show fluctuations potentially attributed to clumps in the stellar wind (Leahy \& Kostka 2008).

We also recall that the interferometric data on Vela X-1 (Choquet et al. 2014), whose supergiant also possesses a strong wind, did not find any differential visibility signatures at the spectral lines above the noise level. GRAVITY commissioning data on this same target also had the same conclusion, even though the signal-to-noise ratio was comparable to the one here (rms in differential visibility amplitudes and phases in the continuum around the $\operatorname{Br} \gamma$ line were $1.2 \%$ and 0.7 , respectively). However, the donor star in Vela $\mathrm{X}-1$ is $\sim 2 \times$ smaller and has a $\sim 5 \times$ smaller mass-loss rate than Wray 977, and the spectral lines in the $K$ band are in absorption or very weak emission.

\subsubsection{Model B: Extended Wind + Gas Stream}

Here we consider the possibility that a gas stream of enhanced density also contributes to the $\operatorname{Br} \gamma$ emission. The manifestation of a gas stream of enhanced density in the hydrogen emission lines of HMXBs is not completely unfamiliar. Yan et al. (2008), e.g., studied the double-peaked $\mathrm{H} \alpha$ emission lines in Cyg X-1, which can be explained by a
P-Cygni-shaped wind profile that follows the orbit of the supergiant as well as emission from a focused stellar wind that has an orbital motion approximately anti-phase to the supergiant. The relevance of the focused wind in $\operatorname{Br} \gamma$ could be even higher than in $\mathrm{H} \alpha$ given that the former line requires much higher densities to be brought into emission.

As alluded to above, a gas stream is predicted to be present in this system from both optical and especially X-ray data. Because of its compactness, a gas stream could also be more efficient than a stellar wind in bringing higher density regions to the outer parts of the system. The simplest stream model would therefore be a binary model consisting of the continuum region at the center and an extra unresolved component. However, it was already shown that a binary model cannot explain the discrepancy between the very small differential visibility phases and the larger differential visibility amplitudes. This is confirmed in a formal binary fit to the data, which is completely unsatisfactory in reproducing both visibility amplitudes and phases simultaneously.

Motivated by this discrepancy, we consider here the possibility that the $\mathrm{Br} \gamma$ line has two emission components: a gas stream of enhanced density, with size on the order of the orbit scale and which accounts for the asymmetric differential visibility phase signatures, and an extended wind, which is symmetric relative to the continuum and accounts for most of the differential visibility amplitude signatures. Because of the lack of higher spectral resolution, it is not possible to perform a spectral decomposition to fix the flux ratios for each component. Because the flux ratios are highly degenerate with the spatial parameters, we fix them to be equal for the stream and wind components. This is motivated by comparing the He I $2.059 \mu \mathrm{m}$ line in Figure 3 for BP Cru and $\zeta^{1}$ Sco: they have similar stellar parameters, so if the extra emission is due to a stream, it would account for roughly $50 \%$ of the line emission. We caution that $\mathrm{Br} \gamma$ and $\mathrm{He}$ I $2.059 \mu \mathrm{m}$ have very different behavior, and the goal of this section is not to provide best-fit parameters, but rather to assess the possibility of a combined wind + gas stream model. Furthermore, we assume that the $\mathrm{Br} \gamma$ emissivity is constant along the stream, which might not be the case. The complex visibility at each spectral channel is therefore modeled as

$$
V(\boldsymbol{u})=\frac{V_{\operatorname{cont}}(\boldsymbol{u})+\frac{f}{2} e^{-\pi^{2}|\boldsymbol{u}|^{2} \frac{\theta_{d}^{2}}{4 \log 2}}+\frac{f}{2} e^{-2 \pi i \sigma_{1} \cdot \boldsymbol{u}}}{1+f},
$$

where all parameters are as in Model $\mathrm{A}$ and $\sigma_{1}$ is the position of the stream. Figure 13 (top) shows the positions of the stream for each wavelength from the best fit to the visibility amplitudes $\left(\chi_{\text {red }}^{2}=2.32\right)$ and differential visibility phases $\left(\chi_{\text {red }}^{2}=1.44\right)$. For convenience, we also show the hypergiant and the four possible predicted positions of the pulsar. Figure 14 shows the resulting size of the extended wind component for each wavelength. The asymmetry in the wind size across wavelength still remains, as in the wind-only model. The wind sizes are slightly increased due to the smaller flux in the wind. The differential phases, on the other hand, are explained by having a compact extra component represented by the gas stream.

For comparison, we also show in Figure 13 (bottom) a stream model in the sky plane. The model follows Leahy \& 

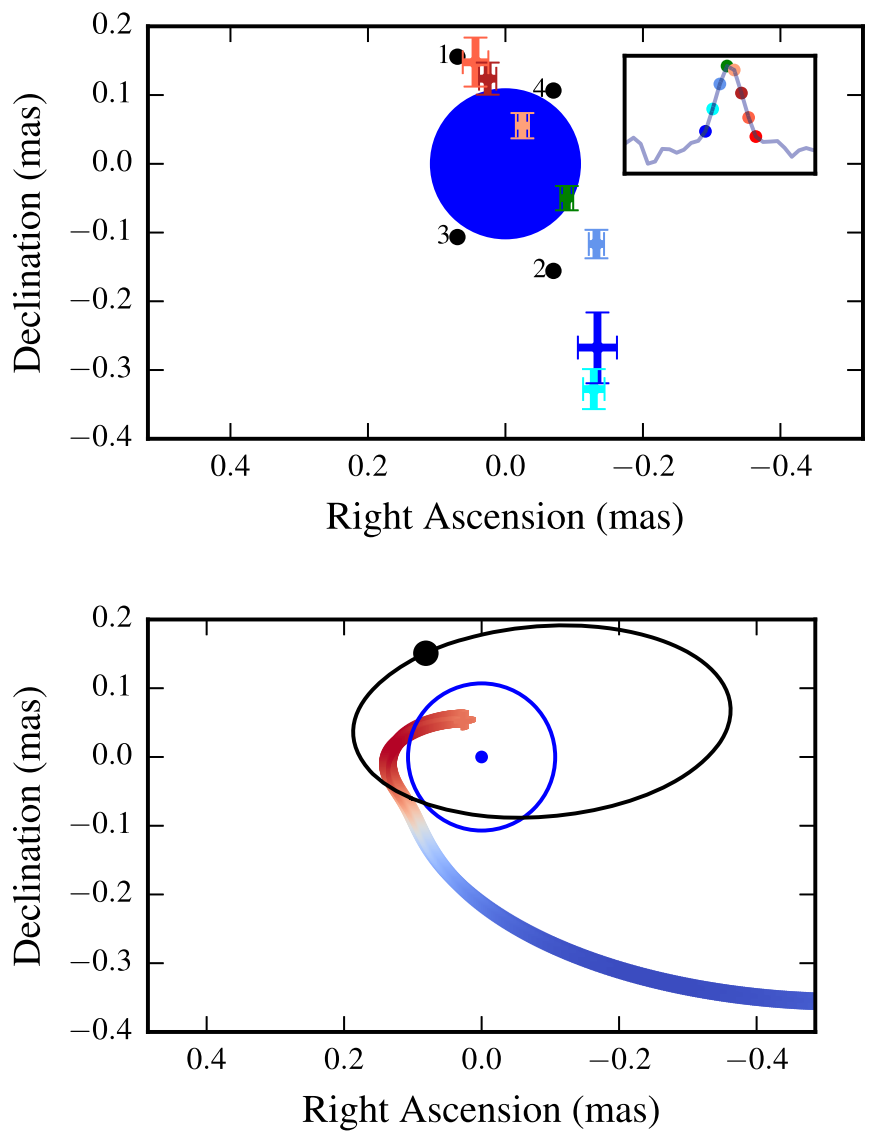

Figure 13. Top: best-fit positions on sky plane for a gas stream in the combined wind + stream model. Also shown are the hypergiant and the four predicted possible positions of the pulsar. Bottom: example of a gas stream model (Leahy \& Kostka 2008) in the sky plane. The colors refer to radial velocities. A gas stream could be an explanation for the asymmetric differential visibility phases across the wavelength.

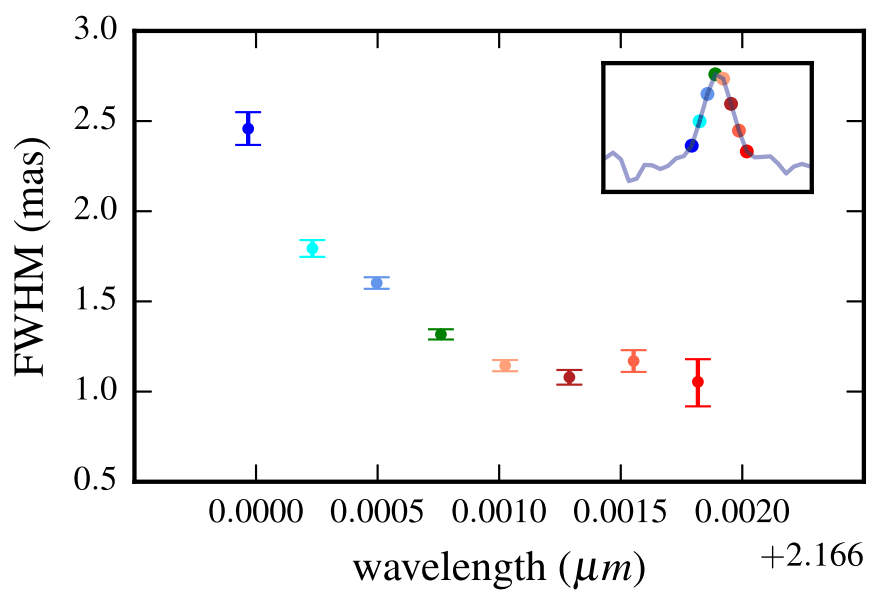

Figure 14. Wind size (FWHM) as a function of wavelength for a combined wind+stream model. The asymmetry in extension across the wavelength remains, as in the wind-only model.

Kostka (2008), and assumes that at each time some mass is ejected from the hypergiant star's surface that intersects the line of centers of the binary. The stream is then formed by propagating each mass element, assuming that the radial velocity follows the CAK wind velocity law and that the angular velocity is given by conservation of angular momentum

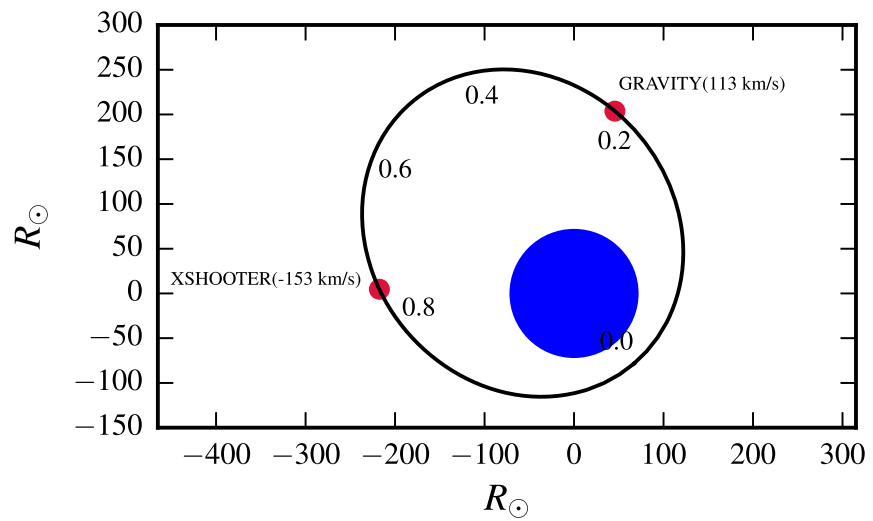

Figure 15. Orbit of the pulsar in the binary plane. Orbital phases are indicated, as well as the positions at the time of observations and the corresponding radial velocity. The donor star is shown in blue with the photospheric radius $\sim 70 R_{\odot}$.

(the hypergiant is rotating). For the model shown, we simply assumed the relevant parameters from Table 1, and that the pulsar is located at position " 1 " $\left(i=60^{\circ} ; \Omega=0^{\circ}\right)$ at the time of observation. The calculation is performed in the binary plane and then projected to the sky plane, with the colors along the stream representing the radial velocity. The stream shape is very sensitive to the assumed parameters, but it could be an explanation for asymmetric differential visibility phases along the emission line.

\section{Additional Data and Future Work}

Here, we present additional spectral data that hint at the next steps in the study of BP Cru with optical interferometry.

As alluded to above, the emission lines in BP Cru may be formed from multiple, distinct components that are either not apparent at the moderate spectral resolution of GRAVITY $(R \sim$ $4000)$ or are modulated by the pulsar's radial velocity curve $\left(v \sim 218 \mathrm{~km} \mathrm{~s}^{-1}\right)$, such as for an accretion disk or possibly a gas stream. This would complicate our model fitting from the previous section.

For these reasons, we have compared the GRAVITY $K$-band spectrum with that measured by XSHOOTER, using archival data $^{16}$ reduced with the publicly available ESO XSHOOTER pipeline. It has a substantially higher spectral resolution $(R \sim$ 11,500) than GRAVITY.

Figure 15 shows the orbit of the pulsar in the binary plane, as well as the positions of the pulsar at the time of the GRAVITY and XSHOOTER observations. The radial velocities of the pulsar are also indicated.

Figure 16 shows the spectra at the $\mathrm{He}$ I $2.059 \mu \mathrm{m}$ and $\mathrm{Br} \gamma$ emission lines for the two instruments. The higher resolution XSHOOTER spectra shows substructure that suggests a more complex line emission, possibly with multiple components. It could therefore be that the line emission has both a contribution from the normal hypergiant wind as well as from a dense gas stream, as is the case for the $\mathrm{H} \alpha$ line in Cygnus X-1 (Yan et al. 2008). We note, in particular, what appears to be a blueshifted $\left(\sim-130 \mathrm{~km} \mathrm{~s}^{-1}\right)$ emission component with $\sim 15 \%$ of the main line strength, when the predicted pulsar radial velocity at the XSHOOTER orbital phase is $-150 \mathrm{~km} \mathrm{~s}^{-1}$. If they indeed trail the pulsar, such components would be redshifted at the time of

\footnotetext{
16 Based on observations with ESO Telescopes at the La Silla Paranal Observatory under programme ID 095.C-0446(A)
} 

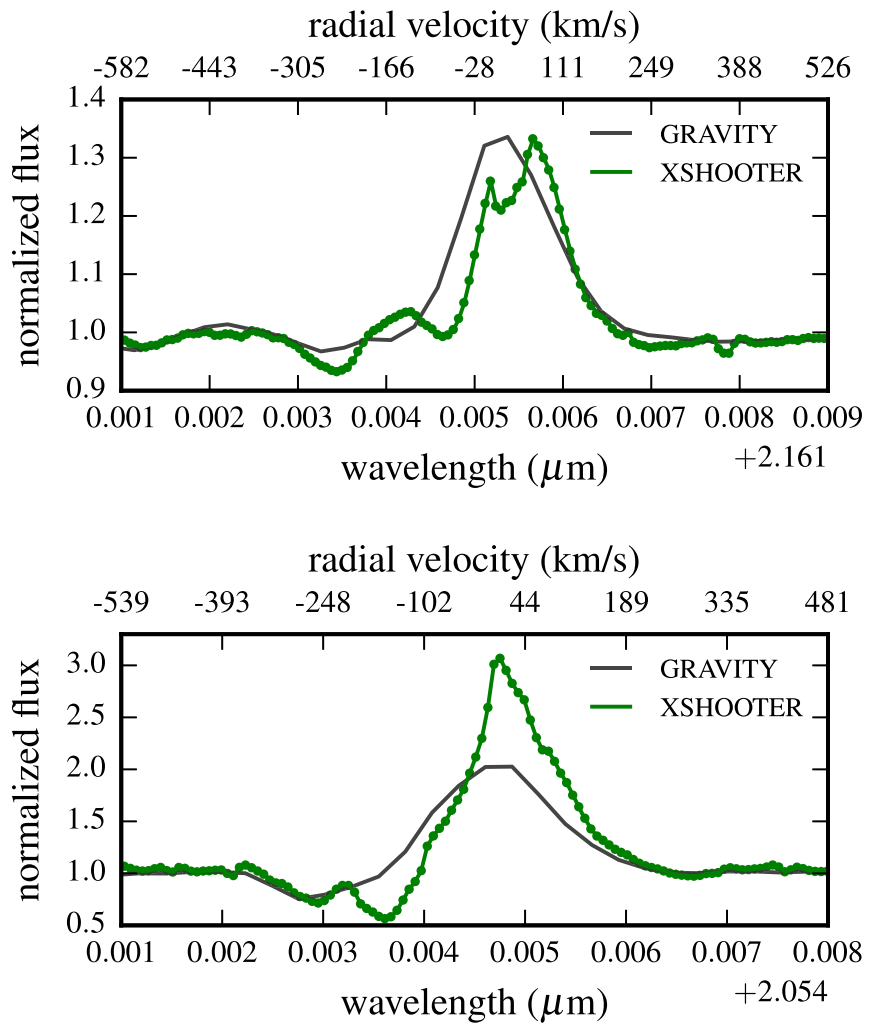

Figure 16. $\mathrm{Br} \gamma$ and $\mathrm{He}$ I $2.059 \mu \mathrm{m}$ lines as seen with GRAVITY UT and XSHOOTER at different orbital phases. The latter has a higher spectral resolution than the former $(R \sim 11,500$ vs. 4000$)$ and shows substructure indicative of multiple line emission components.

the GRAVITY observation and could potentially be related to the interferometric signatures in the red part of the line.

Additional high-resolution spectra at different orbital phases could confirm the presence of such emission components. When coupled with interferometric data, they would also be highly beneficial in testing the different models. Just to mention a few, a comparison between apastron and periastron epochs would help to assess X-ray effects, a comparison between superior and inferior conjunctions could probe the effects of the pulsar at different parts (red versus blue) of the wind, and the wavelength at which the interferometric signatures peak could indicate, with the help of high-resolution spectroscopy, the line emission component that is responsible for the interferometric signatures. All of these could help, for instance, in differentiating between an extended and distorted wind model from a gas stream model or possibly show the need for a combined model.

Finally, we note that the possibility that the differential signatures reported here could be related to the intrinsic variability of the stellar wind of the hypergiant cannot be absolutely excluded with the present data. Differential visibility amplitude and phase signatures have been observed previously in the $\mathrm{H} \alpha$ and $\mathrm{Br} \gamma$ lines of Rigel, a late-B supergiant (Chesneau et al. 2010, 2014). In this case, however, the lines are in absorption and the extension of the wind emission in $\operatorname{Br} \gamma$ is found to originate close to the photosphere $\left(\sim 1.25 R_{*}\right)$, in contrast to the case of BP Cru. Nevertheless, optical spectroscopy monitoring of the isolated early-B hypergiants

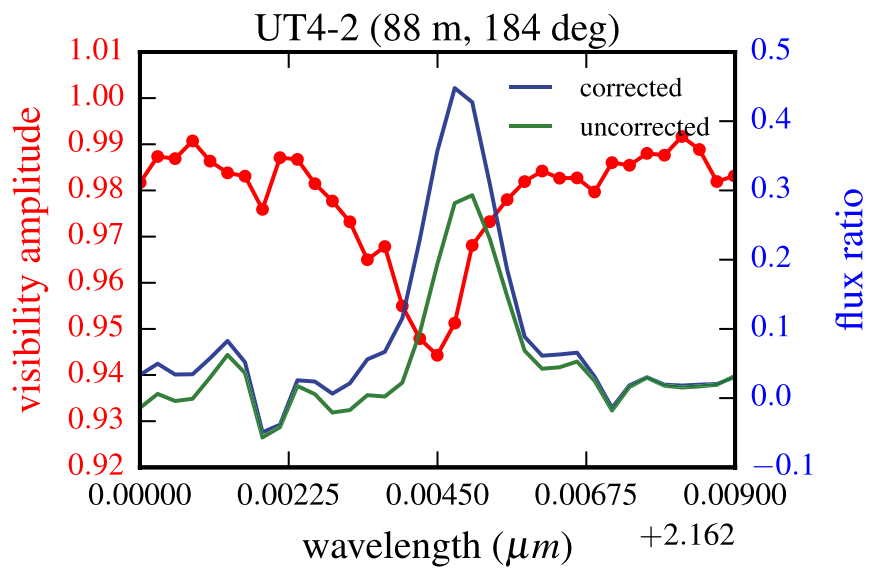

Figure 17. Differential visibility amplitude (red) across the $\operatorname{Br} \gamma$ line for one baseline (red), together with the flux ratio obtained from the spectrum assuming a flat continuum (green) and a continuum that contains a photospheric line (blue). The latter yields a flux ratio $\sim 50 \%$ larger, as well as an increase in the blue portion of the line, in which the interferometric signatures are largest.

mentioned in Section 4 has detected variability in the P-Cygnitype profiles of wind-sensitive lines, in the form of discrete absorption components that could be associated with nonspherical density perturbations (Rivinius et al. 1997). High spectral resolution interferometric observations of such stars would help to assess whether such variability could cause differential signatures of the same scale as that seen in BP Cru, or whether the gravitational and radiation fields of the X-ray pulsar are indeed determinant.

\section{Summary}

We have shown a first analysis of near-infrared interferometric data of the HMXB BP Cru obtained with VLTI/ GRAVITY:

1. The spectrum shows unusual $\mathrm{Br} \gamma$ emission for a star of its spectral type; the higher mass-loss rate may be related to an intrinsically denser wind or, as has been proposed from the X-ray data on this source, to a gas stream of enhanced density.

2 . The continuum visibilities suggest a size $\sim 1 R_{*}$, compatible with the still low infrared excess due to the wind in the $K$ band.

3. Spectral differential interferometry shows differential visibility amplitudes and phases across the $\mathrm{Br} \gamma$ and $\mathrm{He} \mathrm{I}$ $2.059 \mu \mathrm{m}$ emission lines.

4. Any model for the emission lines must produce an asymmetric, extended structure and a smooth spatial centroid gradient with radial velocity.

5. Examples of physically motivated, geometrical models satisfying these constraints include scenarios where the $\mathrm{Br} \gamma$ is dominated by an extended $\left(R \simeq 4-7 R_{*}\right)$, distorted wind or by a combination of extended wind and high-density gas stream.

6. Further orbital phase resolved high-resolution spectroscopy and interferometric observations could help distinguish between models.

To our knowledge, this is the first data set probing HMXB spatial structure on such small microarcsecond scales, in which 
the interaction between the donor star and the pulsar is expected to occur. Follow-up studies may offer the possibility of testing the accretion mechanism and, more generally, the gravitational and radiation effects of the compact object on the stellar environment in these exotic systems.

Based on observations made with ESO Telescopes at the La Silla Paranal Observatory under program ID 60.A-9102. We thank the technical, administrative, and scientific staff of the participating institutes and the ESO Paranal observatory for their extraordinary support during the development, installation, and commissioning of GRAVITY. We also thank F. Martins for providing a stellar atmosphere model of Wray 977. This research has made use of the Jean-Marie Mariotti Center Aspro, OIfits Explorer, and SearchCal services, and of CDS Astronomical Databases SIMBAD and VIZIER.

Software: GRAVITY pipeline (v0.9.6; Lapeyrere Lapeyrere et al. 2014), IRAF, emcee (Foreman-Mackey et al. 2013).

\section{Appendix A}

\section{Pulsar Positions on the Sky Plane}

Here we estimate the predicted pulsar positions in the sky plane (centered on the donor star) at the time of observation based on what is currently known about the system. In addition to the orbital parameters determined from the pulsar's radial velocity curve (Koh et al. 1997), the following parameters are in theory needed:

1. The binary inclination $i$,

2. The mass ratio $q$, and

3. The longitude of the ascending node $\Omega$.

In practice, $q$ is not important because the donor star is much more massive than the pulsar.

We adopt the inclination $i=60^{\circ}$ or $120^{\circ} \pm 10^{\circ}$ from Kaper et al. (2006), which is estimated based on the upper limit on the neutron star mass and the absence of X-ray eclipsing. This allows $a_{X} \approx 0.28$ mas to be estimated from the $a_{X} \sin i$ known from the pulsar's radial velocity amplitude. From the mass ratio $q=\frac{M_{X}}{M_{\mathrm{opt}}} \approx 0.046$ estimated in Kaper et al. (2006) from Wray 977's radial velocity curve, we estimate $a_{\mathrm{opt}}=q a_{X} \approx 0.01$ mas, and therefore the semimajor of the relative orbit $a_{\text {rel }}=a_{X}+a_{\text {opt }} \approx 0.29$ mas $\approx 192 R_{\odot}$. The only remaining parameter to determine is $\Omega$, for which radial velocity measurements are completely independent. However, we may constrain $\Omega$ from X-ray and column density measurements. Kaper et al. (2006) claims that the pulsar is behind Wray 977 in the orbital phase interval $0.18 \lesssim \phi \lesssim 0.34$ based on the decrease in X-ray flux after periastron passage due to absorption by the dense stellar wind, as well as an increase in column density. This allows $\Omega$ to be estimated by setting $x$, the pulsar position in the sky plane, to zero when $\phi \approx 0.26$ :

$$
x \propto \cos \Omega \cos (\omega+\nu)-\sin \Omega \sin (\omega+\nu) \cos i,
$$

where $\nu$ is the true anomaly, which depends on $\phi$ and $e$ only. Plugging in the appropriate values, we get

$$
\tan \Omega \sim \cot (7.85) \cos i \Rightarrow \Omega \sim 0^{\circ} .
$$

Therefore, there are four solutions for the pulsar position, corresponding to $(i, \Omega) \sim\left(60^{\circ}, 0^{\circ}\right),\left(60^{\circ}, 180^{\circ}\right),\left(120^{\circ}, 0^{\circ}\right)$, $\left(120^{\circ}, 180^{\circ}\right)$. They all have the same radial velocity solution and the same orbital phase at superior conjunction, and therefore cannot be distinguished with what is currently known about the system.

Figure 2 shows the four possible positions of the pulsar on the sky plane (centered on Wray 977), along with the six baseline directions.

\section{Appendix B \\ Correcting for the Photospheric Spectrum}

Figure 17 shows the visibility amplitude on top of the flux ratio along the $\mathrm{Br} \gamma$ region for baseline UT4-2, with the flux ratio taken directly from the spectrum by assuming a flat continuum (i.e., continuum $=1$ in the normalized spectrum). Especially on the blue side of the line, it is clear that interferometric signatures occur at regions where the flux ratio is near zero, which is confusing at first. However, one must remember that the unresolved part of the flux (i.e., the "continuum") includes photospheric absorption lines, which get filled by the emission component(s) in the combined spectrum. This is especially clear from the spectra of the comparison stars in Figure 3, which actually show absorption in $\mathrm{Br} \gamma$, likely due to their $\sim 5-10 \times$ smaller mass-loss rate.

Therefore, in order to obtain a more correct value for the flux ratio between the emission component(s) and the unresolved continuum, we must estimate the purely photospheric spectrum of Wray 977. One possibility would be to use stellar atmosphere model codes and set an artificially lower massloss rate. Since this is beyond the scope of this paper, we take a simpler approach and use the spectrum of an isolated blue supergiant star of the same spectral type to estimate the photospheric spectrum. Contrary to the $H$-band Brackett lines, the $\mathrm{Br} \gamma$ line depth is not very sensitive to the star's luminosity/ gravity (Hanson et al. 1996); therefore, the spectrum of a smaller star, with a lower luminosity and much weaker wind, should be a good approximation for the spectrum of Wray's photosphere, at least at the $\mathrm{Br} \gamma$ line.

With this in mind, we chose the star HD 148688 (B1Ia), with the $K$-band spectrum available from Hanson et al. (2005). After degrading the original resolution $(R \sim 12,000)$ to that of GRAVITY, we divide the GRAVITY spectrum by it, resulting in $1+f$, where $f$ is the flux ratio between emission and photosphere. This "photospheric-corrected" flux ratio is also shown in Figure 17.

We note that such a correction ameliorates the presence of interferometric signatures at vanishing flux ratios, as the corrected flux ratio is shifted to the blue (an effect due to the wind emission being slightly redshifted with respect to the photosphere). Additionally, it should be more representative of the true flux ratio. Unfortunately, this method does not work for the He I $2.059 \mu \mathrm{m}$ line, as it is very sensitive to winds and, unlike $\mathrm{Br} \gamma$, goes easily into emission even for this star i.e., its photospheric spectrum is not easily recoverable. 
Appendix C

\section{Spectrum and Interferometric Quantities Full View}
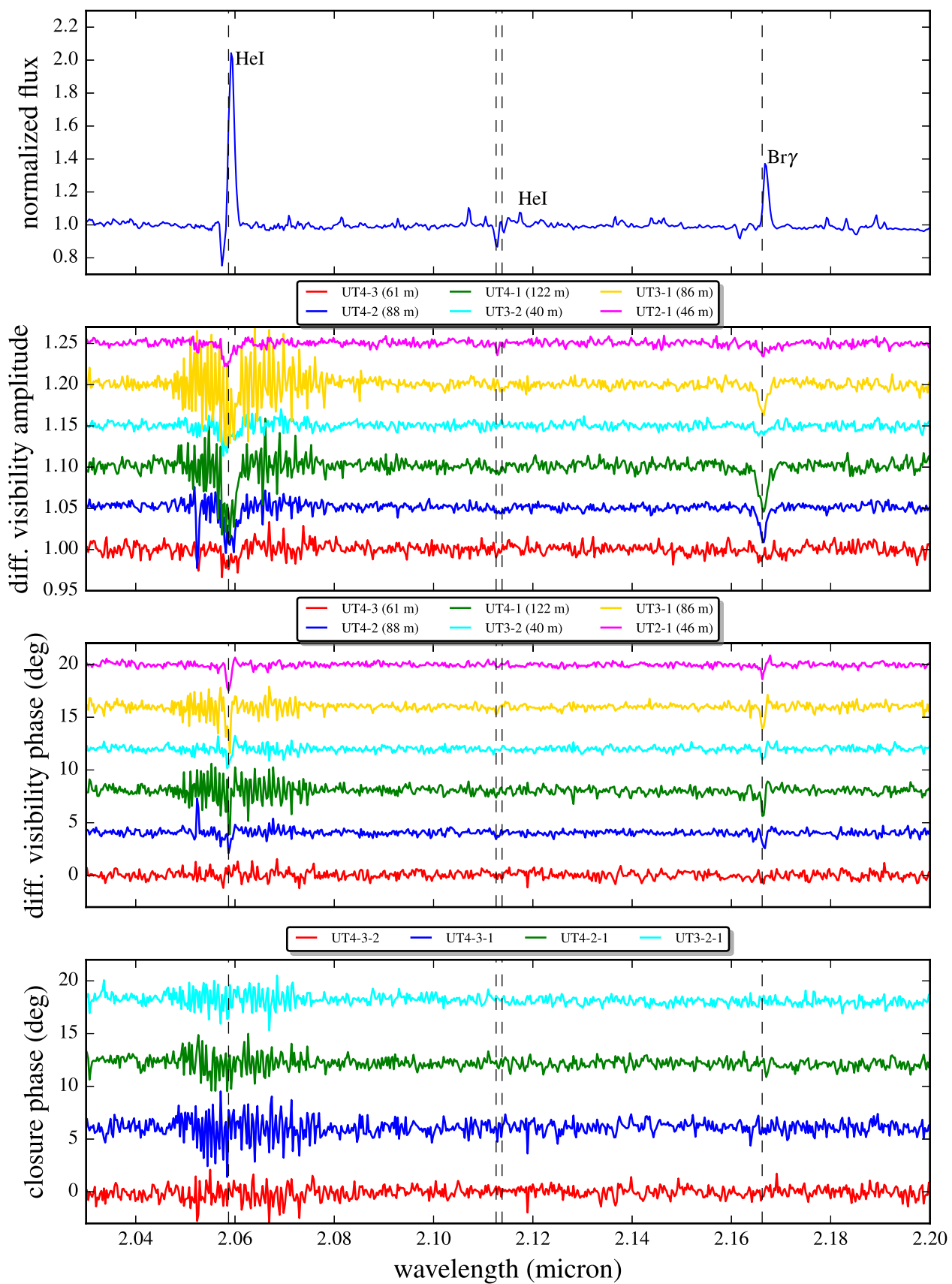

Figure 18. $K$-band spectrum at the original resolution and interferometric quantities. The differential visibility amplitudes, phases, and closure phases have been shifted by $0.05,4^{\circ}$, and $6^{\circ}$ per baseline for plotting. The data have neither been binned nor smoothed.

\section{References}

Bandyopadhyay, R. M., Shahbaz, T., Charles, P. A., \& Naylor, T. 1999, MNRAS, 306, 417

Blondin, J. M. 1994, ApJ, 435, 756

Bondi, H., \& Hoyle, F. 1944, MNRAS, 104, 273

Castor, J. I., Abbott, D. C., \& Klein, R. I. 1975, ApJ, 195, 157

Cechura, J., \& Hadrava, P. 2015, A\&A, 575, A5

Charles, P. A., \& Coe, M. J. 2006, in Compact Stellar X-ray Sources, ed. W. Lewin \& M. van der Klis (Cambridge: Cambridge Univ. Press), 215

Chaty, S. 2011, in ASP Conf. Ser. 447, Evolution of Compact Binaries, ed. L. Schmidtobreick, M. R. Schreiber, \& C. Tappert (San Francisco, CA: ASP), 29
Chesneau, O., Dessart, L., Mourard, D., et al. 2010, A\&A, 521, A5

Chesneau, O., Kaufer, A., Stahl, O., et al. 2014, A\&A, 566, A125

Choquet, É., Kervella, P., Le Bouquin, J.-B., et al. 2014, A\&A, 561, A46

Clark, J. S., Charles, P. A., Clarkson, W. I., \& Coe, M. J. 2003, A\&A, 400, 655

Clark, J. S., Najarro, F., Negueruela, I., et al. 2012, A\&A, 541, A145

Davidson, K., \& Ostriker, J. P. 1973, ApJ, 179, 585

Eisenhauer, F., Perrin, G., Brandner, W., et al. 2011, Msngr, 143, 16

Evangelista, Y., Feroci, M., Costa, E., et al. 2010, ApJ, 708, 1663

Foreman-Mackey, D., Hogg, D. W., Lang, D., \& Goodman, J. 2013, PASP, 125,306

Fuerst, F., Kreykenbohm, I., Kretschmar, P., Ballhausen, R., \& Pottschmidt, K. 2016, ATel, 8870, 1 
Gravity Collaboration, Abuter, R., Accardo, M., et al. 2017, A\&A, 602, A94 Haberl, F. 1991, ApJ, 376, 245

Hanson, M. M., Conti, P. S., \& Rieke, M. J. 1996, ApJS, 107, 281

Hanson, M. M., Kudritzki, R.-P., Kenworthy, M. A., Puls, J., \& Tokunaga, A. T. 2005, ApJS, 161, 154

Islam, N., \& Paul, B. 2014, MNRAS, 441, 2539

Kaper, L., Lamers, H. J. G. L. M., Ruymaekers, E., van den Heuvel, E. P. J., \& Zuiderwijk, E. J. 1995, A\&A, 300 arXiv:astro-ph/9503003

Kaper, L., van der Meer, A., \& Najarro, F. 2006, A\&A, 457, 595

Koh, D. T., Bildsten, L., Chakrabarty, D., et al. 1997, ApJ, 479, 933

Kreykenbohm, I., Wilms, J., Coburn, W., et al. 2004, A\&A, 427, 975

Kudritzki, R.-P., \& Puls, J. 2000, ARA\&A, 38, 613

Lachaume, R. 2003, A\&A, 400, 795

Lapeyrere, V., Kervella, P., Lacour, S., et al. 2014, Proc. SPIE, 9146, 91462D

Leahy, D. A. 1991, MNRAS, 250, 310

Leahy, D. A. 2002, A\&A, 391, 219

Leahy, D. A., \& Kostka, M. 2008, MNRAS, 384, 747

Liu, Q. Z., van Paradijs, J., \& van den Heuvel, E. P. J. 2006, A\&A, 455, 1165

Martínez-Núñez, S., Kretschmar, P., Bozzo, E., et al. 2017, SSRv, arXiv:1701. 08618

Meilland, A., Millour, F., Kanaan, S., et al. 2012, A\&A, 538, A110
Mérand, A., Bordé, P., \& Coudé du Foresto, V. 2005, A\&A, 433, 1155

Monnier, J. D., \& Allen, R. J. 2013, in Planets, Stars and Stellar Systems, ed. T. D. Oswalt \& H. E. Bond (Dordrecht: Springer Verlag), 325

Perez, M. S., \& Blundell, K. M. 2009, MNRAS, 397, 849

Pestalozzi, M., Torkelsson, U., Hobbs, G., \& López-Sánchez, Á. R. 2009, A\&A, 506, L21

Pravdo, S. H., Day, C. S. R., Angelini, L., et al. 1995, ApJ, 454, 872

Rivinius, T., Stahl, O., Wolf, B., et al. 1997, A\&A, 318, 819

Servillat, M., Coleiro, A., Chaty, S., Rahoui, F., \& Zurita Heras, J. A. 2014, ApJ, 797, 114

Shahbaz, T., Bandyopadhyay, R. M., \& Charles, P. A. 1999, A\&A, 346, 82

Stevens, I. R. 1988, MNRAS, 232, 199

Tatulli, E., Millour, F., Chelli, A., et al. 2007, A\&A, 464, 29

Thureau, N. D., Monnier, J. D., Traub, W. A., et al. 2009, MNRAS, 398, 1309

Walder, R., Melzani, M., Folini, D., Winisdoerffer, C., \& Favre, J. M. 2014, in ASP Conf. Ser. 488, 8th Int. Conf. of Numerical Modeling of Space Plasma Flows (ASTRONUM 2013), ed. N. V. Pogorelov, E. Audit, \& G. P. Zank (San Francisco, CA: ASP), 141

Walter, R., Lutovinov, A. A., Bozzo, E., \& Tsygankov, S. S. 2015, A\&ARv, 23, 2

Yan, J., Liu, Q., \& Hadrava, P. 2008, AJ, 136, 631 